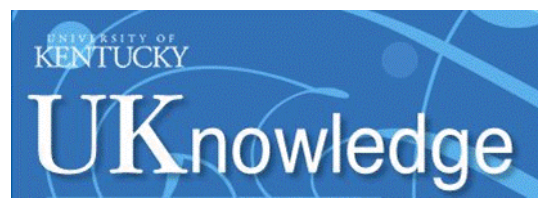

University of Kentucky

UKnowledge

$12-6-2013$

\title{
The Progenitors of the Compact Early-Type Galaxies at High Redshift
}

\author{
Christina C. Williams \\ University of Massachusetts - Amherst \\ Mauro Giavalisco \\ University of Massachusetts - Amherst \\ Paolo Cassata \\ Aix Marseille Université, France \\ Elena Tundo \\ University of Nottingham, U.K. \\ Tommy Wiklind \\ Joint ALMA Observatory, Chile
}

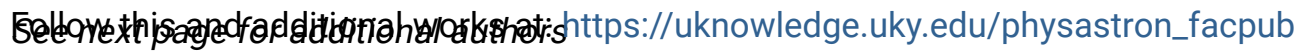

Part of the Astrophysics and Astronomy Commons, and the Physics Commons

Right click to open a feedback form in a new tab to let us know how this document benefits you.

\section{Repository Citation}

Williams, Christina C.; Giavalisco, Mauro; Cassata, Paolo; Tundo, Elena; Wiklind, Tommy; Guo, Yicheng; Lee, Bomee; Barro, Guillermo; Wuyts, Stijn; Bell, Eric F.; Conselice, Christopher J.; Dekel, Avishai; Faber, Sandra M.; Ferguson, Henry C.; Grogin, Norman A.; Hathi, Nimish; Huang, Kuang-Han; Kocevski, Dalibor D.; Koekemoer, Anton M.; Koo, David C.; Ravindranath, Swara; and Salimbeni, Sarah, "The Progenitors of the Compact Early-Type Galaxies at High Redshift" (2013). Physics and Astronomy Faculty Publications. 236. https://uknowledge.uky.edu/physastron_facpub/236

This Article is brought to you for free and open access by the Physics and Astronomy at UKnowledge. It has been accepted for inclusion in Physics and Astronomy Faculty Publications by an authorized administrator of UKnowledge. For more information, please contact UKnowledge@lsv.uky.edu. 


\section{The Progenitors of the Compact Early-Type Galaxies at High Redshift}

\section{Digital Object Identifier (DOI)}

http://dx.doi.org/10.1088/0004-637X/780/1/1

\section{Notes/Citation Information}

Published in The Astrophysical Journal, v. 780, no. 1, p. 1-22.

(c) 2014. The American Astronomical Society. All rights reserved.

The copyright holder has granted permission for posting the article here.

\section{Authors}

Christina C. Williams, Mauro Giavalisco, Paolo Cassata, Elena Tundo, Tommy Wiklind, Yicheng Guo, Bomee Lee, Guillermo Barro, Stijn Wuyts, Eric F. Bell, Christopher J. Conselice, Avishai Dekel, Sandra M. Faber, Henry C. Ferguson, Norman A. Grogin, Nimish Hathi, Kuang-Han Huang, Dalibor D. Kocevski, Anton M. Koekemoer, David C. Koo, Swara Ravindranath, and Sarah Salimbeni 


\title{
THE PROGENITORS OF THE COMPACT EARLY-TYPE GALAXIES AT HIGH REDSHIFT
}

\author{
Christina C. Williams ${ }^{1}$, Mauro Giavalisco ${ }^{1}$, Paolo Cassata ${ }^{2}$, Elena Tundo ${ }^{3}$, Tommy Wiklind ${ }^{4}$, Yicheng Guo $^{5}$, \\ Bomee Lee ${ }^{1}$, Guillermo Barro ${ }^{5}$, Stijn Wuyts ${ }^{6}$, Eric F. Bell ${ }^{7}$, Christopher J. Conselice ${ }^{3}$, Avishai Dekel ${ }^{8}$, \\ Sandra M. Faber ${ }^{5}$, Henry C. Ferguson ${ }^{9}$, Norman Grogin ${ }^{9}$, Nimish Hathi ${ }^{10}$, Kuang-Han Huang ${ }^{11}$, Dale Kocevski ${ }^{12}$, \\ anton Koekemoer ${ }^{9}$, David C. Koo ${ }^{5}$, Swara Ravindranath ${ }^{13}$, and Sara Salimbeni ${ }^{14}$ \\ ${ }^{1}$ Department of Astronomy, University of Massachusetts, 710 North Pleasant Street, Amherst, MA 01003, USA; ccwillia@ astro.umass.edu \\ ${ }^{2}$ Aix Marseille Université, CNRS, LAM (Laboratoire d'Astrophysique de Marseille) UMR 7326, F-13388 Marseille, France \\ ${ }^{3}$ The School of Physics and Astronomy, University of Nottingham, Nottingham NG7 2RD, UK \\ ${ }^{4}$ Joint ALMA Observatory, ESO, Santiago, Chile \\ ${ }^{5}$ UCO/Lick Observatory, Department of Astronomy and Astrophysics, University of California, Santa Cruz, CA 95064, USA \\ ${ }^{6}$ Max-Planck-Institut für Extraterrestrische Physik (MPE), Postfach 1312, D-85741 Garching, Germany \\ ${ }^{7}$ Department of Astronomy, University of Michigan, 500 Church Street, Ann Arbor, MI 48109, USA \\ ${ }^{8}$ Racah Institute of Physics, The Hebrew University, Jerusalem 91904, Israel \\ ${ }^{9}$ Space Telescope Science Institute, 3700 San Martin Boulevard, Baltimore, MD 21218, USA \\ ${ }^{10}$ Carnegie Observatories, Pasadena, CA 91101, USA \\ ${ }^{11}$ Department of Physics and Astronomy, The Johns Hopkins University, 3400 North Charles Street, Baltimore, MD 21218, USA \\ ${ }^{12}$ Department of Physics and Astronomy, University of Kentucky, Lexington, KY 40506, USA \\ ${ }^{13}$ Inter-University of Astronomy \& Astrophysics, Pune, Maharashtra, India \\ ${ }^{14}$ Marlboro College, Marlboro, VT 05344, USA \\ Received 2013 May 30; accepted 2013 October 13; published 2013 December 6
}

\begin{abstract}
We use GOODS and CANDELS images to identify progenitors of massive $\left(M>10^{10} M_{\odot}\right)$ compact early-type galaxies (ETGs) at $z \sim 1.6$. Because merging and accretion increase the size of the stellar component of galaxies, if the progenitors are among known star-forming galaxies, these must be compact themselves. We select candidate progenitors among compact Lyman-break galaxies at $z \sim 3$ on the basis of their mass, star-formation rate (SFR), and central stellar density, and we find that these account for a large fraction of, and possibly all, compact ETGs at $z \sim 1.6$. We find that the average far-UV spectral energy distribution (SED) of the candidates is redder than that of the non-candidates, but the optical and mid-IR SED are the same, implying that the redder UV of the candidates is inconsistent with larger dust obscuration and consistent with more evolved (aging) star formation. This is in line with other evidence suggesting that compactness is a sensitive predictor of passivity among high-redshift massive galaxies. We also find that the light distribution of both the compact ETGs and their candidate progenitors does not show any extended "halos" surrounding the compact "core,"both in individual images and in stacks. We argue that this is generally inconsistent with the morphology of merger remnants, even if gas rich, as predicted by $N$-body simulations. This suggests that the compact ETGs formed via highly dissipative, mostly gaseous accretion of units whose stellar components are very small and undetected in the Hubble Space Telescope images, with their stellar mass assembling in situ, and that they have not experienced any major merging until the epoch of observations at $z \sim 1.6$.
\end{abstract}

Key words: galaxies: elliptical and lenticular, $\mathrm{cD}$ - galaxies: evolution - galaxies: high-redshift - galaxies: star formation - galaxies: structure

Online-only material: color figures

\section{INTRODUCTION}

A generic prediction of the standard cosmological paradigm is that small structures form first, while big ones are assembled later by hierarchical merging. Because the power spectrum is not truncated at any scales relevant to galaxy formation as it evolves, early massive galaxies are comparatively much rarer than galaxies of the same mass that assembled later. These later massive galaxies have assembled their stellar bodies in ways, and over timescales, that are different from the early ones and thus must generally have different properties. Thus, the discovery of old and massive galaxies at high redshift that have rather different structural properties than those of most early-type galaxies (ETGs) in the second half of the Hubble time is interesting because of the possibility it offers to directly explore additional mechanisms of formation of massive galaxies in general and of quenching of the star formation in particular.
Passive galaxies with a large stellar mass, e.g., $M \gtrsim 10^{10} M_{\odot}$, have been identified at redshift as high as $z \sim 3, \approx 16 \%$ of the current age of the universe. A striking characteristic of these young members of the population of ETGs is that they are often very small, up to $\sim$ five times smaller than galaxies of comparable mass in the local universe, and hence have very high stellar density, up to two orders of magnitude higher than local counterparts (see, e.g., Daddi et al. 2005; Trujillo et al. 2006; Bundy et al. 2006; Cimatti et al. 2006; van der Wel et al. 2008; van Dokkum et al. 2008, 2010; Saracco et al. 2009; Bezanson et al. 2009; Damjanov et al. 2009; Williams et al. 2010; Saracco et al. 2010; Cassata et al. 2011, 2013; Weinzirl et al. 2011; Guo et al. 2012; Ryan et al. 2012; Lee et al. 2013).

At $z \sim 1.6$, the population of ETGs is dominated by the compact ones, with more than $\gtrsim 80 \%$ being smaller than the lower $1 \sigma$ of local ETGs of the same mass (Cassata et al. 2011, 2013). In the local universe, these compact ETGs seem 
to be exceedingly rare, although there is still ongoing debate on whether this apparent paucity is real or the result of bias in local surveys such as the Sloan Digital Sky Survey (SDSS; Bournaud et al. 2007; Hopkins et al. 2009a; Taylor et al. 2010; McLure et al. 2013; Ragone-Figueroa \& Granato 2011; Oogi \& Habe 2013; Nipoti et al. 2012).

Given this apparent spectacular evolution, it is no surprise that much effort went into exploring the possible evolutionary mechanisms that have driven it. For example, it has been suggested that individual compact ellipticals might form extended stellar halos either by in situ star formation or by dry merging and accretion (Naab et al. 2007; van Dokkum et al. 2010; Whitaker et al. 2010; Nipoti et al. 2012; Oser et al. 2012). In particular, it has also been proposed that interactions and repeated minor merging events, even if they do not increase the stellar mass by a large amount, can energize the innermost stellar orbits and "puff up" the compact galaxies (Newman et al. 2012). Concurrently, the size evolution of the population of ETGs as a whole can also be driven by the addition of new members coming from the late quenching of massive, large galaxies (Valentinuzzi et al. 2010a, 2010b; Cassata et al. 2011; Newman et al. 2012; Poggianti et al. 2013; Carollo et al. 2013). From the analysis of the evolution of ETGs of different stellar densities as a function of redshift, Cassata et al. (2013) conclude that the addition of new, larger ETGs is required to explain the overall increase in their numbers from $z \sim 1$ to the present (Ilbert et al. 2010; Pozzetti et al. 2010). In any case, it is important to realize that an accurate census of compact galaxies in the local universe is still missing because the SDSS samples are very likely biased against such systems (Scranton et al. 2002; Valentinuzzi et al. 2010a; Cassata et al. 2013; Carollo et al. 2013) and also because the descendants of the compact galaxies might not be easily recognized in the local universe if they became the core of systems that developed extended stellar halos (e.g., Kormendy et al. 2009; Nipoti et al. 2012; Huang et al. 2013b).

Regardless of the problem of their subsequent evolution, however, it seems clear that compact ETGs were very abundant at high redshift and that they largely dominate the population of passive galaxies at redshift $z \sim 1.2-2.8$ (Cassata et al. 2013), at least at large mass $\left(M>10^{10} M_{\odot}\right)$. This raises the question of how such massive systems could form in such a relatively short time. There are indications that whatever process is responsible for quenching star formation in massive galaxies is largely controlled by the star-formation rate (SFR), with more actively star-forming galaxies being more likely to quench (e.g., Peng et al. 2010, 2012), and that more compact systems are more likely to quench more effectively than are those with more diffuse mass distribution (Bell et al. 2012; Cheung et al. 2012; Lee et al. 2013). However, even before they quench, the problem of how massive galaxies with such high stellar density could form and why they dominate the population of massive passive galaxies at high redshift is interesting because it seems to imply a specific formation mechanism different from other galaxies. Is the physics that shuts off star formation in high-redshift galaxies producing only compact remnants as a byproduct? Or, does it preferentially affect those galaxies with high stellar densities? One popular mechanism to both shut off the star formation in a galaxy and also produce spheroidal morphologies are major mergers (Barnes 1992; Hernquist 1992, 1993; Springel et al. 2005). Evidence of this mechanism is seen in the local universe (Sanders et al. 1988; Sanders \& Mirabel 1996), and evidence of merging has been observed out to high redshift (Lotz et al. 2008; Robaina et al. 2010; Kartaltepe et al. 2010, 2012). During a merging event, however, a substantial fraction of the preexisting stars of the merging galaxies are dispersed to larger radii. It is therefore difficult to produce compact remnants unless the progenitors themselves are also very compact, and in any case the fraction of stars scattered to large radii is not negligible (Ostriker 1980; Naab et al. 2007, 2009). Gas-rich mergers may produce remnants with a very compact core through in situ star formation because of the highly dissipative nature of the gas (Khochfar \& Silk 2006; Hopkins et al. 2008; Wuyts et al. 2010; Bournaud et al. 2011), but the preexisting stars are still dispersed to large radii and the gas fractions must be high (e.g., $\gtrsim 60 \%-70 \%$ ) in order to produce a large fraction of the stellar mass in a compact remnant (Hopkins et al. 2008, 2009b; Wuyts et al. 2010). Although it is possible that current data have not yet probed the low surface brightness regions surrounding compact ETGs to the sensitivity required in order to rule out evidence of major merger activity, tidal debris, or dispersed stars, there is some general evidence that these galaxies are truly compact in size, with no diffuse or extended structure surrounding them (van Dokkum et al. 2008; Szomoru et al. 2012; Bezanson et al. 2009). Much debate still exists on the role of major merging in the buildup of the stellar mass of massive galaxies in general, regardless of whether it is compact, (Bell et al. 2006; Robaina et al. 2010; Genel et al. 2010; Williams et al. 2011b; LópezSanjuan et al. 2012; Conselice et al. 2013) and in particular for morphologically selected spheroidal galaxies (Bundy et al. 2007, 2009; Kaviraj et al. 2013a, 2013b).

Theoretical work have shown that violent disk instability (VDI; Dekel et al. 2009a), driven by intense accretion of cold gas from the cosmic web (Birnboim \& Dekel 2003; Kereš et al. 2005, 2009; Dekel \& Birnboim 2006; Dekel et al. 2009b), can lead to the formation of compact massive galaxies. The gas-rich disks are Toomre unstable, with large-scale transient perturbations and massive bound clumps. The mutual interactions between these perturbations exert torques that drive angular momentum out and mass in, in the form of clump migration and gas inflow in the inter-clump disk medium (Bournaud et al. 2011; Dekel et al. 2013; Forbes et al. 2013). As long as the inflow rate is more rapid than the SFR in the disk, the inflow to the center is gas rich, and the product is compact (Dekel \& Burkert 2013). The induced central starburst can eventually lead to quenching, by gas consumption into stars (Diamond-Stanic et al. 2012), by outflows driven by stellar or AGN feedback (Springel et al. 2005), or by morphological quenching (Martig et al. 2009). The star-formation quenching may also be related to the shutting off of the cold gas supply. Theory, confirmed by simulations, predicts that after $z \sim 2$, for dark matter halos with masses of $\sim 10^{12} M_{\odot}$ and above, the incoming gas is heated by a virial shock that can be supported because of the long cooling times (Dekel \& Birnboim 2006).

The extent to which these processes affect the formation of compact ETGs at $z>2$ remains unconstrained. Thus, progress is likely to come from the identification and empirical studies of their progenitors before and while they quench, namely while they are still in the star-forming phase and when they shut it down. Some have proposed potential progenitors based on matching the stellar mass and the volume density of the ETGs with those of star-forming galaxies together with assumptions of possible evolutionary paths (Whitaker et al. 2012; Barro et al. 2013; Patel et al. 2013; Stefanon et al. 2013). Stefanon et al. (2013) in particular have identified progenitors of the most massive compact ETGs $\left(M>10^{11} M_{\odot}\right)$ among the most massive $\left(M>10^{10.6} M_{\odot}\right)$ galaxies at $z>3$ by 
projecting their observed stellar masses and SFRs assuming various star-formation histories (SFHs). Regardless of whether the morphological properties, SFRs, and stellar mass of the more general population of putative progenitors were consistent with the compactness of their descendants among all passive galaxies at $z \sim 2$ and their specific SFRs have not been considered in detail, something we set to do here.

In this paper, we try to answer the following question. Because we do not know of any physical mechanism capable of transforming a non-compact stellar system into a compact one, do star-forming galaxies exist at suitably high redshifts (i.e., such that there is time for quenching) that are plausible progenitors of compact and massive ETGs? Namely, are there plausible progenitors with stellar mass and SFR such that they can plausibly explain their descendants at $\langle z\rangle \sim 1.6$ ? To answer this question, we identify star-forming galaxies at $z \sim 3$ that, if they quench, can reproduce both the mass and the stellar density, and hence size, of the observed compact galaxies at $\langle z\rangle \sim 1$.6. In other words, we try to see whether we can identify the progenitors on the basis of the evolutionary consistency assuming only that (1) the star-forming galaxies quench early enough to be passive at $z \sim 1.6$ and (2) that no (unknown) physical mechanism transforms non-compact stellar systems into compact ones. With a sample of plausible progenitors, we then can compare their properties to those of other star-forming galaxies that are not plausible progenitors and see whether there are differences that might offer some insight into the formation of the ETGs. We present the sample, and its selection, in Sections 2 and 3. In Section 4, we study the properties of these plausible progenitors and compare them with the rest of the normal star-forming galaxy population at $z>3$. We also investigate the distribution of stellar populations of different ages in the galaxies. In Section 5, we discuss the implications of our results for the evolution of compact ETGs in the context of the evolutionary drivers and quenching mechanisms affecting these galaxies. Throughout this paper, we assume a cosmology with $\Omega_{\Lambda}=0.7$, $\Omega_{m}=0.3$, and $H_{o}=70 \mathrm{~km} \mathrm{~s}^{-1} \mathrm{Mpc}^{-1}$.

\section{DATA}

\subsection{Multi-wavelength Imaging and Photometry}

In this paper, we use data from the Great Observatories Origins Deep Survey (GOODS; Giavalisco et al. 2004), and 4 epoch depth observations with HST/WFC3 from the Cosmic Assembly Near-infrared Deep Extragalactic Legacy Survey (CANDELS). This covers $113 \mathrm{arcmin}^{2}$ of the GOODS-South field, which includes the CANDELS Deep region (Grogin et al. 2011; Koekemoer et al. 2011) and the early release science (ERS) (Windhorst et al. 2011). The $H$-band images in the 4 epoch deep and ERS regions reach $1 \sigma$ fluctuations of 26.6 and 26.3 $\mathrm{AB} \operatorname{arcsec}^{-2}$, respectively.

In total we make use of panchromatic photometry in GOODSSouth, including $U$-band data from the Visible Multiobject Spectrograph (VIMOS) on the Very Large Telescope (VLT; Nonino et al. 2009); $H S T / A C S B, V, i, z$-band, HST/WFC3 $J$, $H$-band, and VLT/ISAAC $K_{s}$ photometry (Retzlaff et al. 2010); Spitzer/IRAC 3.6, 4.5, and $5.7 \mu \mathrm{m}$ imaging (M. Dickinson et al., in preparation), Spitzer/MIPS $24 \mu \mathrm{m}$ imaging (M. Dickinson et al., in preparation), and GOODS-Herschel/PACS $100 \mu \mathrm{m}$ imaging (Elbaz et al. 2011).

We measure photometry (in IRAC channels and blue-ward) for galaxies in the 4 epoch CANDELS data using the object template-fitting method (TFIT; Laidler et al. 2007) software

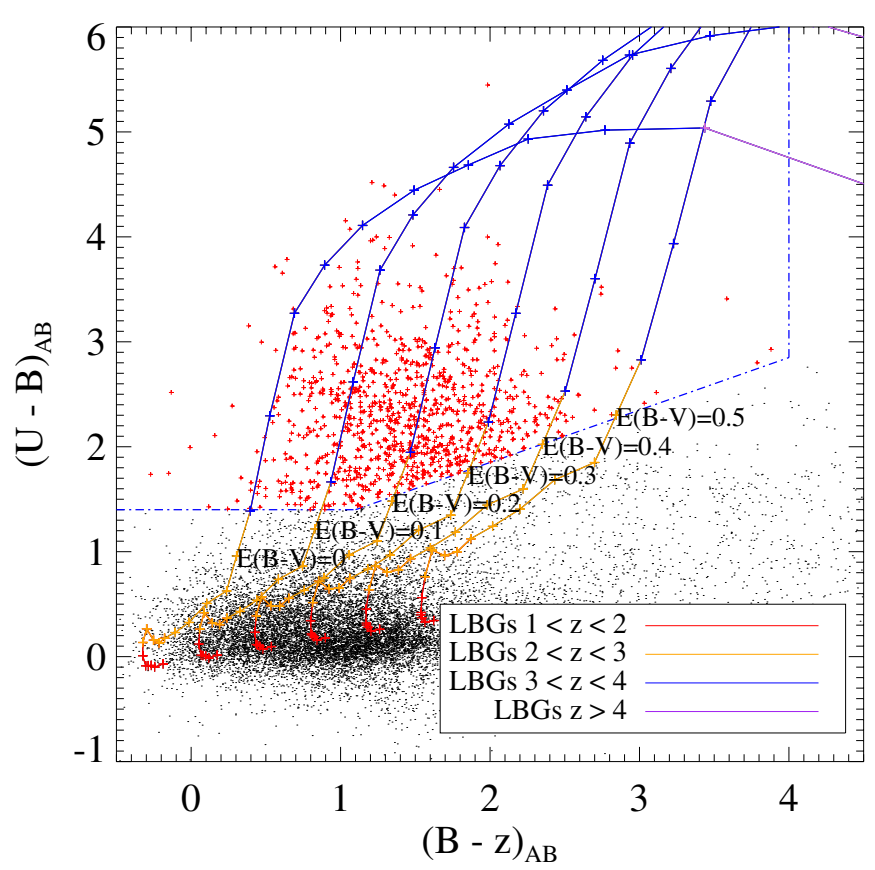

Figure 1. LBG color selection. Generic LBG SED tracks (see the text) plotted with varying $E(B-V)$.

(A color version of this figure is available in the online journal.)

package, which allows us to construct spectral energy distributions (SEDs) with mixed-resolution data sets. All details about the construction of the multi-wavelength photometry constructed using TFIT is discussed in Guo et al. (2013).

\subsection{LBG Sample Selection}

The colors and ages of the high-redshift ETGs are such that they should be star forming at $z \sim 3$ (Daddi et al. 2005; Kaviraj et al. 2013a; Onodera et al. 2012). Therefore, we select star forming galaxies at redshift $z \sim 3$ from the ACS $z$-band imaging using the Lyman-break color selection (Steidel \& Hamilton 1993; Giavalisco 2002), including $z$-band detections with $z \gtrsim 26.5$ (AB magnitudes). The ACS $z$ band is $\sim 90 \%$ complete down to 26.5 for galaxies with size less than 0.3 arcsec in half-light radius (Huang et al. 2013a). Our $U$-band dropout selection criteria are

$$
\begin{aligned}
& \left(U_{\text {VIMOS }}-B_{435}\right) \geqslant 0.85+0.5 \times\left(B_{435}-z_{850}\right) \wedge \\
& \left(U_{\text {VIMOS }}-B_{435}\right) \geqslant 1.4 \wedge B_{435}-z_{850} \leqslant 4,
\end{aligned}
$$

where $\wedge$ refers to the logical "and" operator. We also require signal to noise a of at least three in the $B_{435}$ and $z_{850}$ bands to ensure robust color measurements. We calibrate our $U$-band dropout selection using redshifted, continuously star-forming stellar population synthesis models of Bruzual \& Charlot (2003) with varying values of dust extinction, following Calzetti et al. (2000). The Lyman break galaxy (LBG) selection, with these varying stellar population models, are shown in Figure 1, and includes 943 objects. We also remove from the LBG sample those galaxies without unique WFC3 $\mathrm{H}$-band detections $(36 \%$ of the original sample), and those with photometric redshifts of less than two (an additional 14\%), to ensure only the most robust sample of $z>3$ galaxies are used in the following analysis. Our final sample of LBGs includes 517 galaxies, 180 of which are $H<25$, and all of which have $z \leqslant 26.5$. As we see later, a sample of candidate progenitors of compact massive ETGs 


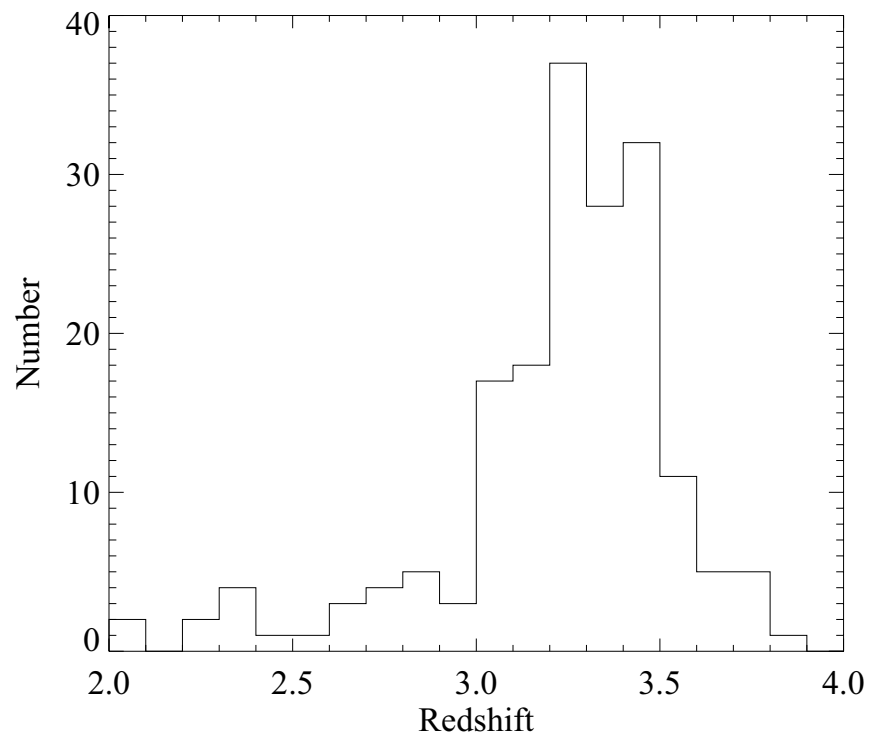

Figure 2. Redshift distribution for $H<25$ LBGs.

will be selected among the most compact LBG which, given the sensitivity of the GOODS images, is $\gtrsim 85 \%-90 \%$ complete down to $z \leqslant 26.5$. The redshift distribution for $H<25$ LBGs is shown in Figure 2.

\subsection{Measuring Physical Properties of LBGs}

We measure photometric redshifts and stellar masses for our sample of LBGs by fitting stellar population synthesis models to their observed SEDs. Photometric redshifts are derived using PEGASE 2.0 (Fioc \& Rocca-Volmerange 1997), where we integrate the probability distribution function of redshift to derive the photometric redshift. Of our LBGs, $20 \%$ have spectroscopically confirmed redshifts (Cristiani et al. 2000; Le Fèvre et al. 2004; Szokoly et al. 2004; Vanzella et al. 2005, 2008; Mignoli et al. 2005; Ravikumar et al. 2007; Kurk et al. 2009; Popesso et al. 2009; D. Stern et al., in preparation). Using these photometric redshifts (or spectroscopic where available), stellar masses are derived through fitting the stellar population synthesis models of Bruzual (2007) with a Salpeter initial mass function, as described in Guo et al. (2012). The models also use the Calzetti dust extinction law (Calzetti et al. 2000) and the Madau (1995) cosmic opacity, and a number of SFHs including exponentially decreasing ( $\tau$-models with varying timescale $\tau$ ), constant, and twocomponent (delay) models composed of linearly increasing and exponentially decreasing components (e.g., Lee et al. 2010). While we found that there generally is good quantitative agreement between the stellar mass derived using these three SFHs, we ended up adopting the exponentially declining or constant SFH that minimizes the $\chi^{2}$.

We measure SFRs of our LBGs using the observed slope of the rest-frame ultraviolet (UV) SED, i.e., we do not use the SFR derived from the SED fitting procedure. We make use of the correlation between the dust obscuration and the slope of the rest-frame UV SED of starburst galaxies (Meurer et al. 1999) to derive the dust-corrected UV luminosity and subsequently the dust-corrected UV SFR using the conversion factor by Madau et al. (1998).

Morphological measurements in the $H$ band (restframeoptical at $z \sim 3$ ) of our LBGs are performed using the GALFIT package (Peng et al. 2002). Nearby objects are masked using segmentation maps produced by SExtractor in the same configuration as that used for the initial source detection. We fit light profiles using a Sersic model. We use a point spread function (PSF) constructed from an average of unsaturated stars. To ensure robust morphological measurements, we remove galaxies from our sample if GALFIT indicates the morphological measurement is questionable, namely such that we can not confidently rule out that they are stars, or the signal to noise in the $H$-band photometry is less than 15 . Previous investigations of GALFIT measurements with low signal to noise data indicate that a signal to noise of at least 10 is required to produce unbiased measurements (Ravindranath et al. 2006; Trujillo et al. 2007; Cimatti et al. 2008). These criteria remove 5\% of our LBG sample, two LBGs on the basis of GALFIT error, and eight LBGs on the basis of low signal to noise in the $H$ band.

Our study uses a measure of the circularized half-light radius, defined as $R_{e}=r_{e} \sqrt{b / a}$, where $r_{e}$ is the length of the semimajor axis in arcseconds and $b / a$ is the axis ratio of the galaxy. All measurements of the circularized half-light radius are converted to $\mathrm{kpc}$ using the photometric redshift of the object (or spectroscopic redshift if available). The uncertainty on the physical size of each galaxy is taken to be $20 \%$ of the measurement, on the basis of the simulations by Cassata et al. (2011), as the error returned by GALFIT does not include systematic errors.

\section{IDENTIFICATION OF PLAUSIBLE PROGENITORS OF COMPACT ETGS}

Our goal in this section is to see whether among star-forming galaxies at redshift $z \sim 3$, e.g., $U$-band dropout LBGs, there are plausible progenitors of the compact ETGs at $\langle z\rangle \sim 1.6$.

The redshift range between $z \sim 3$ and 1.6 encompasses about 2 Gyr, so essentially every LBG at $z \sim 3$ that quenches its star formation quickly enough after the epoch of the observation will satisfy our definition of a "passive galaxy." Although quenching is an obvious necessary condition to be classified as passive, it is not sufficient because not every quenched star-forming galaxy will have (1) the stellar mass, (2) the specific SFR, and (3) the stellar density of the compact, massive, and passive galaxies that we are considering here, i.e., those selected using the criteria by Cassata et al. (2013). Thus, to identify the likely progenitors of this specific group of galaxies, let's first define exactly what we mean by massive, passive, compact galaxies.

We use the same criteria adopted by Cassata et al. (2011, 2013) to define their sample. Specifically, galaxies were selected to be in the redshift range $1.2<z<2.8$ by means of photometric redshift (or spectroscopic when available), and for having stellar mass $M^{*}>10^{10} M_{\odot}$ and specific star-formation rate $\log _{10}(\mathrm{sSFR})<-2 \mathrm{Gyr}^{-1}$. In the case of the ETG sample, both stellar mass and sSFR have been estimated from SED fitting to stellar population synthesis models, assuming the $\mathrm{SFH}$ is an exponentially declining one, which is appropriate for the case of galaxies that are completing the cessation of their starformation activity. We note that our requirement of passivity is a relatively stringent one, with the sSFR limit being about $1 / 10$ of the current value of the Milky Way. Recent selections of passive galaxies in the literature use sSFR thresholds that are an order of magnitude or even higher than our criteria (e.g., Barro et al. 2013). This is required, in our opinion, to clearly distinguish a passive galaxy, like present-day ellipticals, from relatively low-level but continuous star formation, such as that of 
massive disks, because the SFHs of these two types of galaxies are very different.

Compact galaxies are defined in terms of their size and stellar mass, hence the average stellar-mass surface density within the effective (half-light) radius $r_{e}$, namely $\Sigma_{50}=\left(0.5 M_{*} / \pi r_{e}^{2}\right)$. To be classified as compact, passive galaxies of a given mass must have a stellar-mass surface density larger than the value that exceeds the $1-\sigma$ scatter of the stellar mass-size relationship for local $(z=0)$ ETGs of the same mass (thus, by definition $\approx 17 \%$ of local ETG are compact). Ultra-compact galaxies are those that are 0.4 dex smaller than the $1-\sigma$ value. In terms of projected density, the local mass-size relationship is roughly parallel to the line of constant $\Sigma_{50}$, so that the aforementioned definitions translate into the following conditions: $\Sigma_{50} \gtrsim 3 \times 10^{9} M_{\odot} \mathrm{kpc}^{-2}$ for compact galaxies and $\Sigma_{50} \gtrsim 1.2 \times 10^{10} M_{\odot} \mathrm{kpc}^{-2}$ for ultracompact galaxies (see Cassata et al. 2011). We adopt these more general classifications in the remainder of this study.

The sample by Cassata et al. (2013) includes a total of 107 ETGs with stellar mass $M>10^{10} M_{\odot}$ in the range of $1.2<$ $z<2.8$, with average redshift $\bar{z}=1.6$, of which 76 are compact according to the aforementioned definition, 42 of which are also ultra-compact, and the remaining 31 are normal ETGs, i.e., within the $1 \sigma$ scatter in the mass-size relationship observed at $z=0$. At high-redshift, ultra-compact galaxies appear to dominate the population of massive, passive galaxies. Of the 21 galaxies of this sample that have $z \geqslant 2,4$ are normal, 3 are compact, and 14 are ultra-compact, suggesting that compact and ultracompact galaxies dominate the population of passive galaxies at high redshift and thus were the first to become passive.

\subsection{Consistent Star-formation Histories}

As the mass-size distributions of galaxies in the top panel of Figure 3 show, the stellar masses of the LBGs are significantly smaller than those of the passive ones. Can the progenitors of the compact ETGs be found among them then? The LBGs are star-forming galaxies and thus continue to increase their stellar mass until they quench. Thus, the question can be reformulated as whether there is enough time between $z \sim 3$ and $z \sim 1.6$ for the LBGs to cessate their star-formation activity, reach an sSFR low enough to satisfy the aforementioned definition of passivity, and build up enough stellar mass to reproduce the distribution of the ETGs. In the Appendix, we discuss possible quenching paths that the LBGs need to follow to be classified as passive at $z \sim 1.6$ according to our definition. To summarize, from the knowledge of the stellar mass and the SFR of the individual galaxies at the time of the observation and assuming a functional form for the SFH during the quenching phase, we can predict the final stellar mass and SSFR of the former LBG once they have quenched. This calculation shows that there are indeed plausible quenching scenarios that could evolve some of the LBGs in our sample into passive galaxies as defined earlier. Because the quenching phase is believed to be fairly rapid (e.g., Peng et al. 2012), in the calculation we model it with a decreasing exponential function $\exp (-t / \tau)$, with the timescale $\tau$ equal to $100 \mathrm{Myr}$ for all galaxies. This timescale was estimated using the sound-crossing time in compact galaxies (we used $v_{s}=20 \mathrm{~km} \mathrm{~s}^{-1}$ for the sound speed in the interstellar medium (ISM) of LBGs, as discussed in more detail in the Appendix). We also assumed that the declined phase of the SFR began at the time of observation, i.e., $t_{q}=t_{\text {obs }}$.

As discussed in the Appendix, the time difference between $z \sim 3$ and $z \sim 1.6$ is sufficiently large that from the point of view of the selection of the candidate progenitors, these are
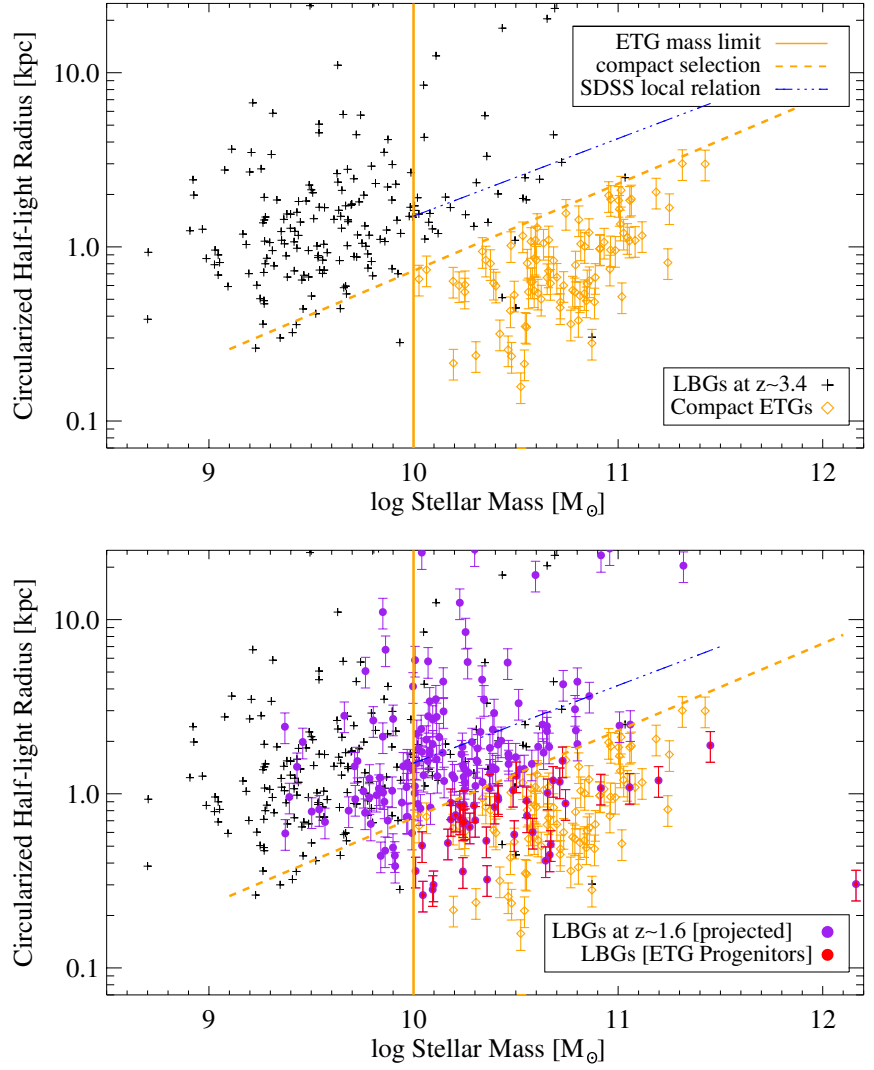

Figure 3. Top panel: observed size-mass relationship of LBGs (black crosses), compared with that of the compact ETG sample of Cassata et al. (2013), along with ETG mass selection (solid orange line) and compactness selection (orange dashed line). The blue dot-dashed line indicates the local mass-size relation of ETGs from the SDSS (Cassata et al. 2013). Bottom panel: as the top panel, with additional points indicating the projected mass-size distribution of LBGs after they satisfy the condition of passivity, namely $\log _{10} \mathrm{sSFR}<-2 \mathrm{Gyr}^{-1}$ (purple and red points). The stellar mass of LBGs increases according to the assumed SFH and no size evolution (i.e., each projected purple and red point has a corresponding black cross at the same half-light radius. See the text for details). Candidate ETG progenitors are selected from this sample (red) as those LBGs whose projected mass puts them in the compact ETG selection window. (A color version of this figure is available in the online journal.)

conservative assumptions, since both a later quenching time $t_{q}$ and a longer $\tau$ would still result in galaxies that are passive according to our criterion while yielding larger stellar mass and hence increasing the number of candidates.

\subsection{Progenitor Morphologies}

Comparing only the two stellar-mass distributions of descendants and candidate progenitors (after they have finished forming stars) is not sufficient to set up physically motivated selection criteria. A key property to consider when trying to identify the progenitors of compact ETGs is the morphology of these systems, and specifically their very high stellar density. The question we are posing is as follows. Can star-forming galaxies at $z \sim 3$ with any morphology be the progenitors of the compact passive ones? Or only galaxies with certain morphology can evolve into such systems, either via merging or via in situ star formation?

On the basis of $N$-body simulations of binary merging events, Wuyts et al. (2010) suggested that the compact ETG progenitors are to be searched for among star-forming galaxies that are compact themselves and also have large gas fractions, i.e., $f_{\text {gas }} \gtrsim 60 \%$. These progenitors, in turn, can form most of the 
stellar mass of the final descendant in a highly concentrated merger-triggered burst. Barro et al. (2013) also argue that a powerful nuclear starburst in a merger remnant producing most of the stellar mass of the remnant itself will result in a galaxy that, once passive, will resemble the compact ones. As we will discuss later, however, it is reasonable to believe that whether most of the final stellar mass is formed during a merger-induced nuclear burst or an in situ star formation, the progenitors must be compact themselves, have stellar densities comparable to or higher than the descendants, and have similar stellar-mass profiles. In other words, it is not reasonable to expect that starforming galaxies at $z>2$ whose light profile is more diffuse than the $z \sim 1.6$ compact passive ones can be their progenitors, even if most of the stellar mass of the final descendant is produced after the epoch of observation in a compact region. This is true both for the case of a single galaxy that forms stars and quenches star formation in situ, or for galaxies that merge.

The physical reason is that an existing diffuse stellar component cannot be shrunk into a compact one and it cannot be hidden from observations either, regardless of whether or not a new, more massive compact stellar component is subsequently created (note that mergers puff up compact structures into diffuse ones, not vice versa). To first order, if a diffuse component is observed in the CANDELS $H$-band images in a star-forming galaxy at $z \sim 3$, the same diffuse component will also be observed in the same image if the same galaxy were placed at $z \sim 1.6$ after quenching its star-formation activity. This is in part because of the lower redshift and in part because of the increase in stellar mass between the time when the galaxy is observed and when the quenching process is complete.

\subsection{Progenitor Selection}

Given that the quenching phase is not instantaneous, candidate progenitors must have (1) smaller stellar mass than the passive ones, but SFRs such that after they quench, the final stellar mass density reproduces that of the passive ones; and (2) morphology and size similar to that of the ETGs, since the ongoing formation of stars does not change the dynamics of the preexisting stellar orbits and hence the appearance of the galaxies. Here we will explore a scenario where these progenitors essentially evolve at constant size by converting gas into stars while their stellar mass and density increases, therefore maintaining the compact light profile observed in the compact ETG.

It is important to understand that we are not postulating that all galaxies that satisfy the two aforementioned general requirements will evolve by growing their stellar mass at essentially constant size. On average, galaxies evolve by growing their stellar mass and enlarging their size, as shown by the existence of a mass-size relationship and the overall size evolution of star-forming galaxies (e.g., Ferguson et al. 2004; Hathi et al. 2008b; Nagy et al. 2011; Huang et al. 2013a). However, not all galaxies must do so, or must all follow the same mass-size growth path, as evidenced by the scatter in the mass-size relationship itself. Nor are we saying that those LBGs that satisfy the two aforementioned points quench their star formation after the epoch of observation and appear passive (as per our definition) at $\langle z\rangle \sim 1.6$. What we are saying is that if LBGs at $z \sim 3$ include progenitors of the compact ETGs at $\langle z\rangle \sim 1.6$, then the morphological properties of the latter imply that the former must grow in mass at essentially constant size, and have stellar mass and SFRs such that under general assumptions about their SFHs subsequent to the epoch of observation, they quench and are passive at the epoch when the compact ETGs are observed.

To identify such LBGs, if they exist, we must model the quenching of their star formation activity. Thus, we need to assume (1) the time when they begin quenching and (2) how they quench, i.e., the form of the declining SFH. The quenching phase can start at any time after the epoch of observation of the LBG, or even slightly before, since a galaxy in the early phase of declining star formation would still be classified as a "Lyman-break galaxy" as long as this phase is not too advanced. The details of SFHs of galaxies during the quenching phase are not known (Lee et al. 2011), and there are suggestions that star-forming galaxies can have bursty and chaotic SFHs (Lee et al. 2012). Therefore, a simple function such as an exponential decay is almost certainly an oversimplification, especially on short timescales. If the goal is to model the formation of ETGs, however, all that is relevant is that the SFR overall decreases on a relatively long timescale, namely long compared with the timescale of rapid bursts (i.e., a few $10^{7} \mathrm{yr}$ ), since their red colors (at the time of observation), high masses, and very low sSFRs imply that they must have been actively star forming at least 1 Gyr earlier (Onodera et al. 2012). Previous studies investigating the SFHs of the compact ETGs have supported this interpretation (Cimatti et al. 2008; Saracco et al. 2011, 2012; Kaviraj et al. 2013a; Daddi et al. 2005).

It is important to keep in mind that the decaying exponential function that we used to model the quenching phase of the LBGs' star-forming activity has nothing to do with the function adopted to describe their SFHs up to the time of the observation. The latter is used to model the assembly of the galaxies that we observe and only to estimate their stellar mass at the time of observation (recall that we tested both an exponentially declining and a delayed increasing function when measuring the observed stellar mass, with similar results). To describe the $\mathrm{SFH}$ of the quenching phase, which begins at or after the time of observation, we use only the exponentially declining SFH with $\tau=100 \mathrm{Myr}$ as described in the Appendix, and this is used to estimate the additional stellar mass produced after the time of observation and during the quenching phase (we do not make an attempt to incorporate mass loss from stars). This stellar mass is then added to the mass of the LBG formed up to the time of observation to derive the projected mass distribution of the candidate progenitors of the compact ETGs and compare with the observed one for compact ETGs (see Figures 3 and 4). In any case, the combination of the time when they start quenching and the duration of the quenching phase must be such that by redshift $\sim 1.6$ the galaxies would be observed as passive, according to the aforementioned criteria, and that the stellar-mass distribution must reproduce that of the compact ETGs.

Thus, once we assume the starting time of the quenching phase and the timescale $\tau$ (e.g., the limiting case of the soundcrossing time, $\tau \approx 100 \mathrm{Myr}$ ) from the measured (photometric) redshift, SFR, and stellar mass of the LBG, we can estimate the redshift at which the galaxy will satisfy the condition for passivity, as well as its stellar mass at that time. Also, from the measured size of the LBG and under the assumption that the galaxy evolves at constant size, we can estimate the stellar density when the galaxy is passive and see whether it matches the projected stellar density of the compact ETGs, i.e., $\Sigma_{50}>3 \times 10^{9} M_{\odot} \mathrm{kpc}^{-2}$.

We present our compact progenitor selection in Figure 3, which in the top panel shows the mass-size relationship for the 

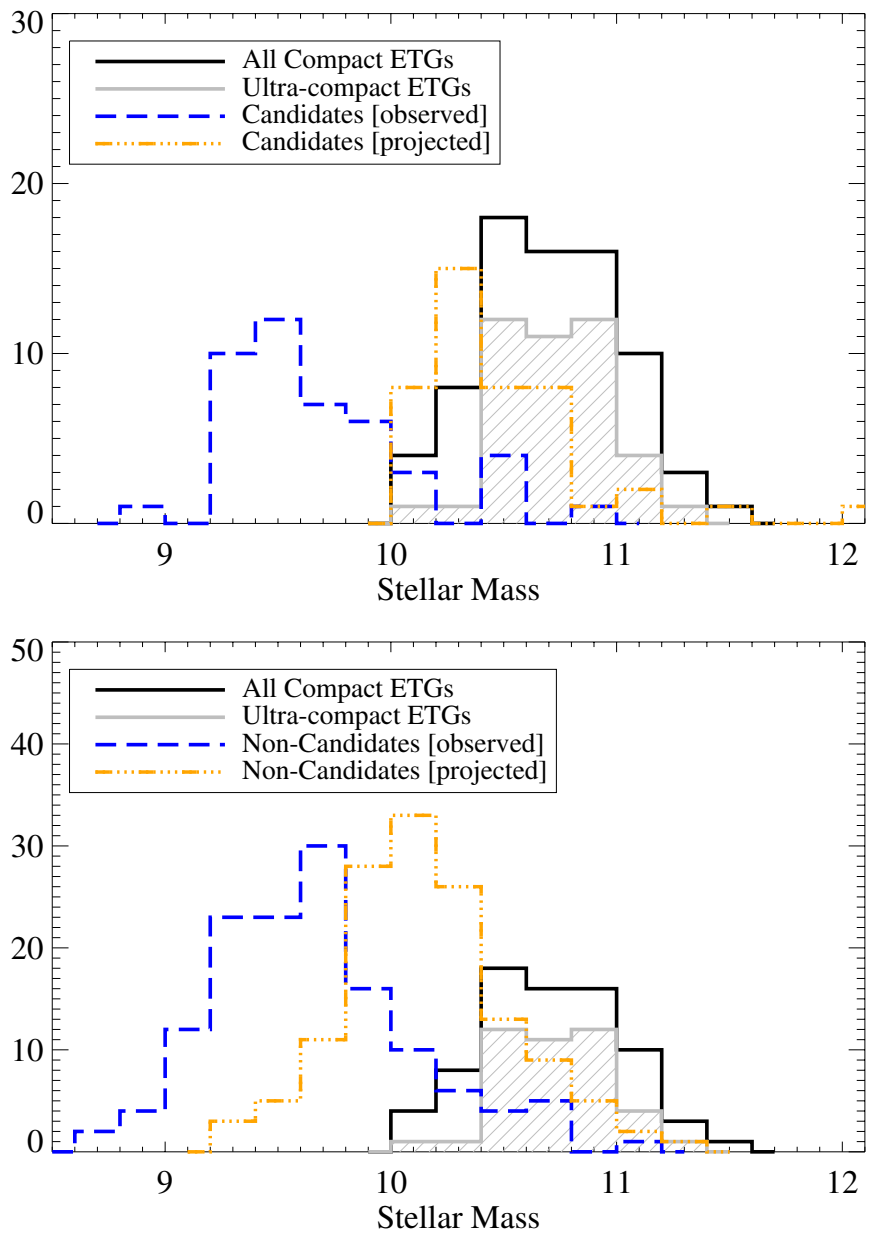

Figure 4. Distribution of observed stellar mass and projected stellar mass (projected using the assumed SFH outlined in the text), for the candidate LBG sample (top panel) and non-candidate LBGs (bottom panel). For comparison, the mass distributions of all compact ETGs and the ultra-compact sub-sample are shown in black and gray, respectively.

(A color version of this figure is available in the online journal.)

LBGs as observed, and also the Cassata et al. (2013) ETG sample and its selection criteria. In the bottom panel, we also plot where the same LBGs will lie in the mass-size diagram assuming their projected stellar mass, meaning their expected mass after they satisfy the condition of passivity, namely sSFR $<-2 \mathrm{Gyr}^{-1}$. Each LBG accumulates stellar mass according to its observed SFR and assumed SFH. The distribution of final projected mass compared with the observed mass is shown in Figure 5, and it should be noted that a large fraction of the projected final mass of most galaxies must be extrapolated using the observed SFR and assumed SFH. Those 44 LBGs with projected properties that meet our compact ETG selection criteria are our sample of candidate compact ETG progenitors. The rest of the LBGs (136), which end up either less massive or with lower stellar density, are non-candidates. We also note in this figure the existence of 11 LBGs from our sample that are already compact in stellar density, as observed, at $z \sim 3$.

As might be expected, the candidate plausible progenitors tend to have higher SFRs than the non-candidates. This trend can be seen in the SFR-M* relation for the two samples, which is shown in Figure 6. There is one galaxy in the candidate sample for which we have estimated a rather large SFR $\left(\sim 10^{4}\right)$. We have investigated the SED of this galaxy for any signature of AGN

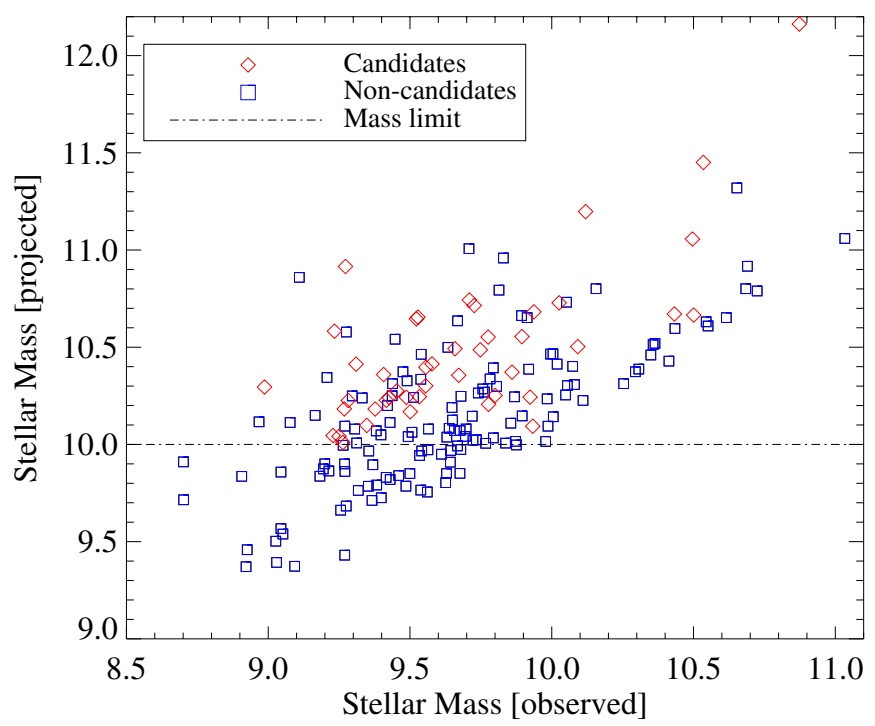

Figure 5. Distribution of projected stellar mass (calculated with the assumed SFH outlined in the text), as a function of observed stellar mass, for the candidate LBG sample (red diamonds) and non-candidate LBGs (blue squares). The dot-dashed line indicates the ETG mass selection. Non-candidate LBGs that lie above this line are too extended to be selected as progenitors, and below this line, non-candidates do not meet the mass selection.

(A color version of this figure is available in the online journal.)
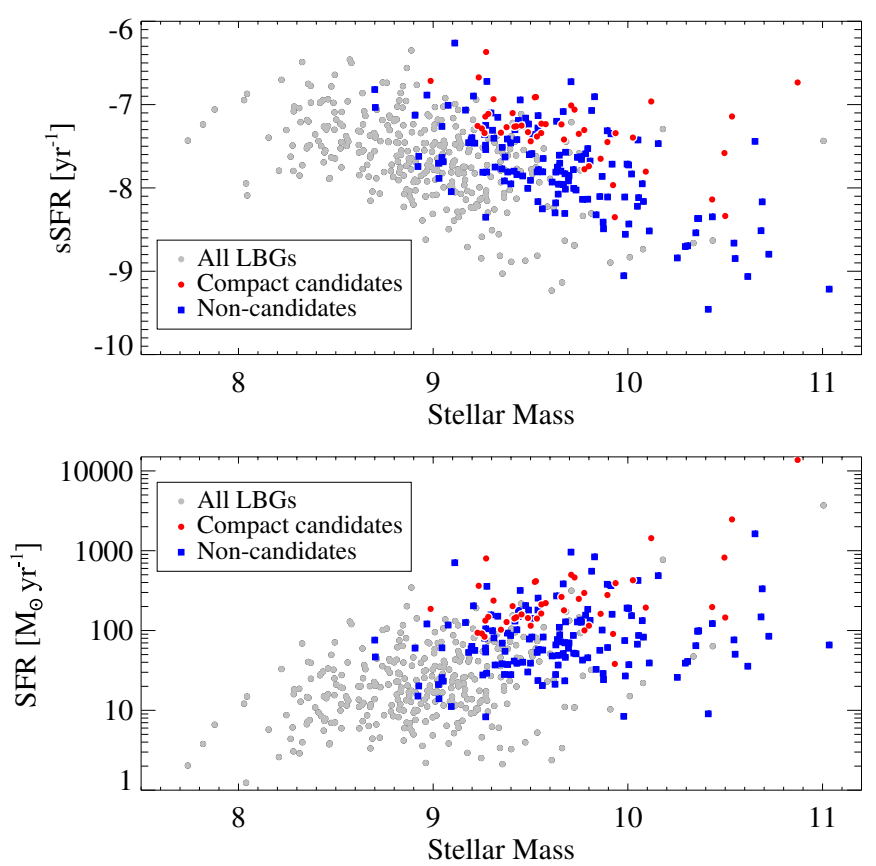

Figure 6. Bottom panel: SFR versus mass distribution for candidate LBGs (red), non-candidate LBGs (blue), and compared with all LBGs selected from Figure 1 (gray) including $H>25$ objects. Top panel: sSFR versus mass for the same galaxies.

(A color version of this figure is available in the online journal.)

and found that it is not detected at $24 \mu \mathrm{m}$, nor does it have an $\mathrm{X}$-ray detection in the Chandra 4Ms image. This galaxy appears to simply be one of the redder galaxies in our candidate LBG sample, hence its high SFR. Even if our de-reddening procedure overestimates the SFR of this object, which we think is quite likely, this galaxy will regardless end up in the candidate sample because its high stellar mass and compact size already places it in the candidate selection window. Its exclusion, or inclusion, does 

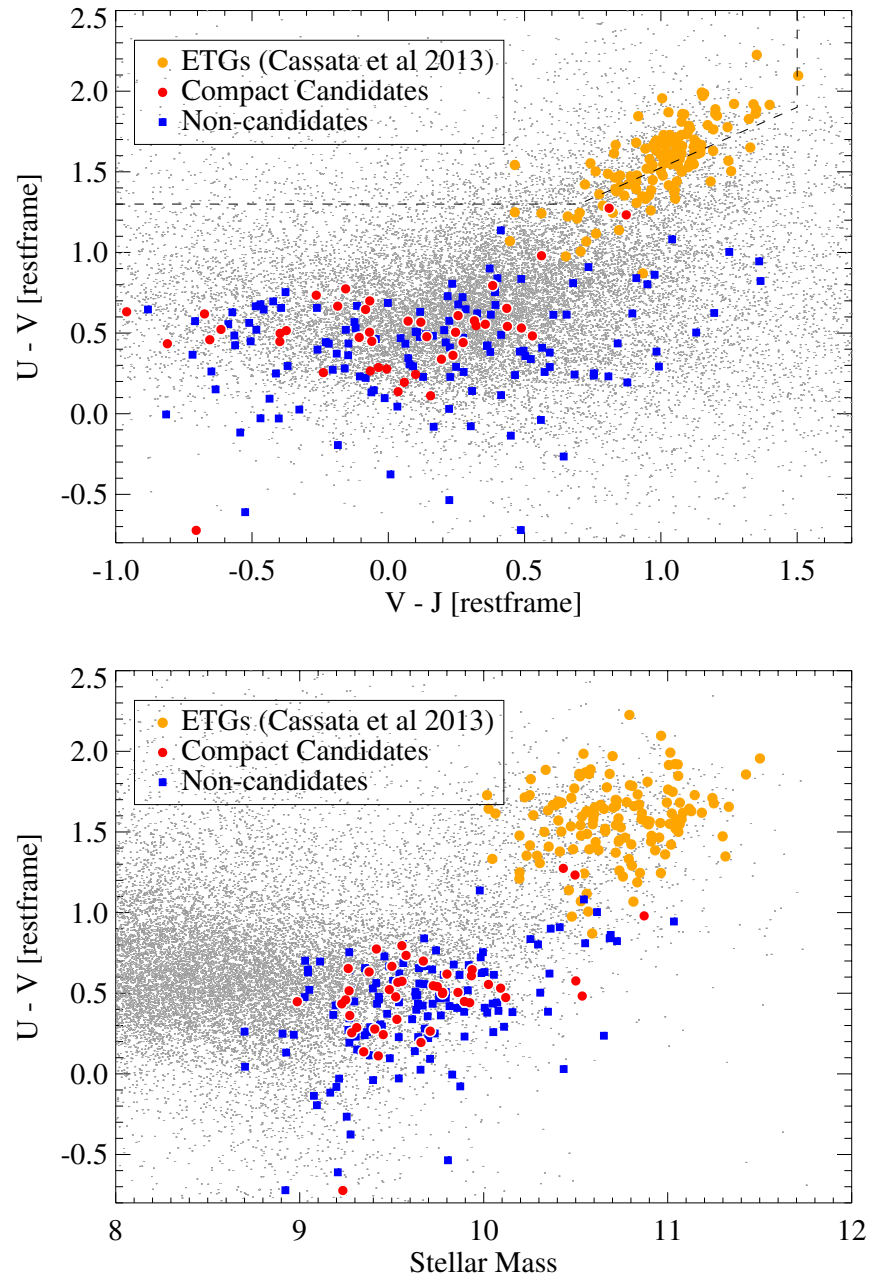

Figure 7. Top panel: restframe $U-V$ versus $V-J$ colors of the high-redshift ETG sample of Cassata et al. (2013, red points) compared with those of the candidate (orange) and non-candidate (blue) LBG samples. The dashed line indicates the red sequence in $U V J$ color space as defined by Williams et al. (2011b). Bottom panel: $U-V$ color versus stellar mass diagram for the same galaxies. The candidate LBG sample tends to have redder $U-V$ color distribution while occupying a similar range in $V-J$ to the non-candidates. Their stellar-mass distributions are roughly similar.

(A color version of this figure is available in the online journal.)

not significantly change the results presented in the following sections. We also note that the candidates do not differ in their observed mass distribution from the non-candidates. This can be further seen in their color-mass diagram for the samples considered here, shown in Figure 7. We also present in Figure 7 the rest-frame $U-V$ versus $V-J$ color-color diagram, showing the colors of the three samples of star-forming and passive galaxies. The color distributions are important for understanding inherent differences in the two samples of LBGs, as we will show in Section 4.

\section{RESULTS}

\subsection{Properties of Compact Progenitors}

\subsubsection{SEDs}

As mentioned in Section 2, we have fit stellar population synthesis models (Bruzual 2007) to each galaxy. The bestfitting SED template to each galaxy, found as explained in Section 2, has been used to generate an average SED tem- plate for candidate and non-candidate samples. We restrained ourselves to those LBGs in both samples that have spectroscopically confirmed redshifts ( $\sim 25 \%$ of all LBGs, $\sim 44 \%$ of candidates, $\sim 18 \%$ of non-candidates), as this will minimize the diluting effects of including objects with only photometric redshifts that have larger uncertainties. We have also derived average empirical SEDs directly from the observed photometry, $\mathrm{k}$-corrected to common restframe wavelength using the best fit templates. The errors on the average empirical SED are estimated using the following procedure. With each galaxy's observed photometry, we produce a Gaussian deviate of each photometric measurement, given that point's photometric error. We then re-calculate the average empirical SED. We do this procedure 10,000 times, and the standard deviation of the average empirical SEDs of the Gaussian deviates is the error on the average.

These two measures of the average SEDs for the two samples of spectroscopic LBGs are shown in the top panel of Figure 8. We have chosen to normalize the average SEDs at $5000 \mathrm{~A}$, the vicinity of the observed $K_{s}$ band, so as to emphasize differences in the UV and optical parts of the SED. At redshift $\sim 3$, there are four prominent emission lines characteristic of star-forming galaxies that may enter the $K_{s}$ band (or also the $H$ band, $H_{\alpha}, H_{\beta}$, $\mathrm{O}$ II, and $\mathrm{O}$ III), and in a large fraction of our galaxies, we do see an enhancement of the flux density in the $K_{s}$ and/or $H$ band, relative to the best fitting SED template. Therefore, we choose to normalize by the value of the best-fitting template at $5000 \mathrm{~A}$ rather than bias the normalization high by using the observed flux density in the $K_{s}$ band.

The excess flux density of these contaminated photometric points with respect to other photometric points and the best-fit SED suggests the photometry of some galaxies is affected by the presence of emission lines. We investigate the extent to which these lines may affect the average SEDs in the bottom panel of Figure 8 by repeating the analysis after removing the individual affected photometric point from the galaxy's observed SED if one of the four lines listed earlier enters any bandpass at greater than $1 \%$ of the maximum transmittance of the band. The result of this test is shown in the bottom panel of Figure 8. It is clear that removing contaminated photometry brings the points in the average empirical SED that are based on the observed $K_{S^{-}}$ and $H$-band photometry into better agreement with the average SED template. The difference between the SEDs of the compact candidates and non-candidates is still clear when removing the affected photometry.

The results shown in Figure 8 indicate that, on average, the compact candidates with spectroscopic redshifts have a redder restframe-UV SED than the non-candidates, but an otherwise apparently identical optical one. There are two explanations for a redder UV slope: an older population of stars (e.g., a more evolved burst of star formation) or a larger amount of dust obscuration (on average). The effects of age or dust on galaxy SEDs are generally degenerate and are notorious for confusing the measurement of physical properties of galaxies. However, we argue here that the flatness and consistency of the average SEDs of candidates and non-candidates red-ward of $4000 \mathrm{~A}$ argue in favor of the interpretation that the difference is due to a difference in average stellar ages (since the peak of SF activity), and not average dust properties.

Figure 9 illustrates how the UV and optical parts of the Bruzual \& Charlot (2003) SED templates of star-forming galaxies vary with varying age, varying dust obscuration, and varying both age and tau for constant $\mathrm{t} / \tau(t / \tau \propto \mathrm{sSFR})$. The 

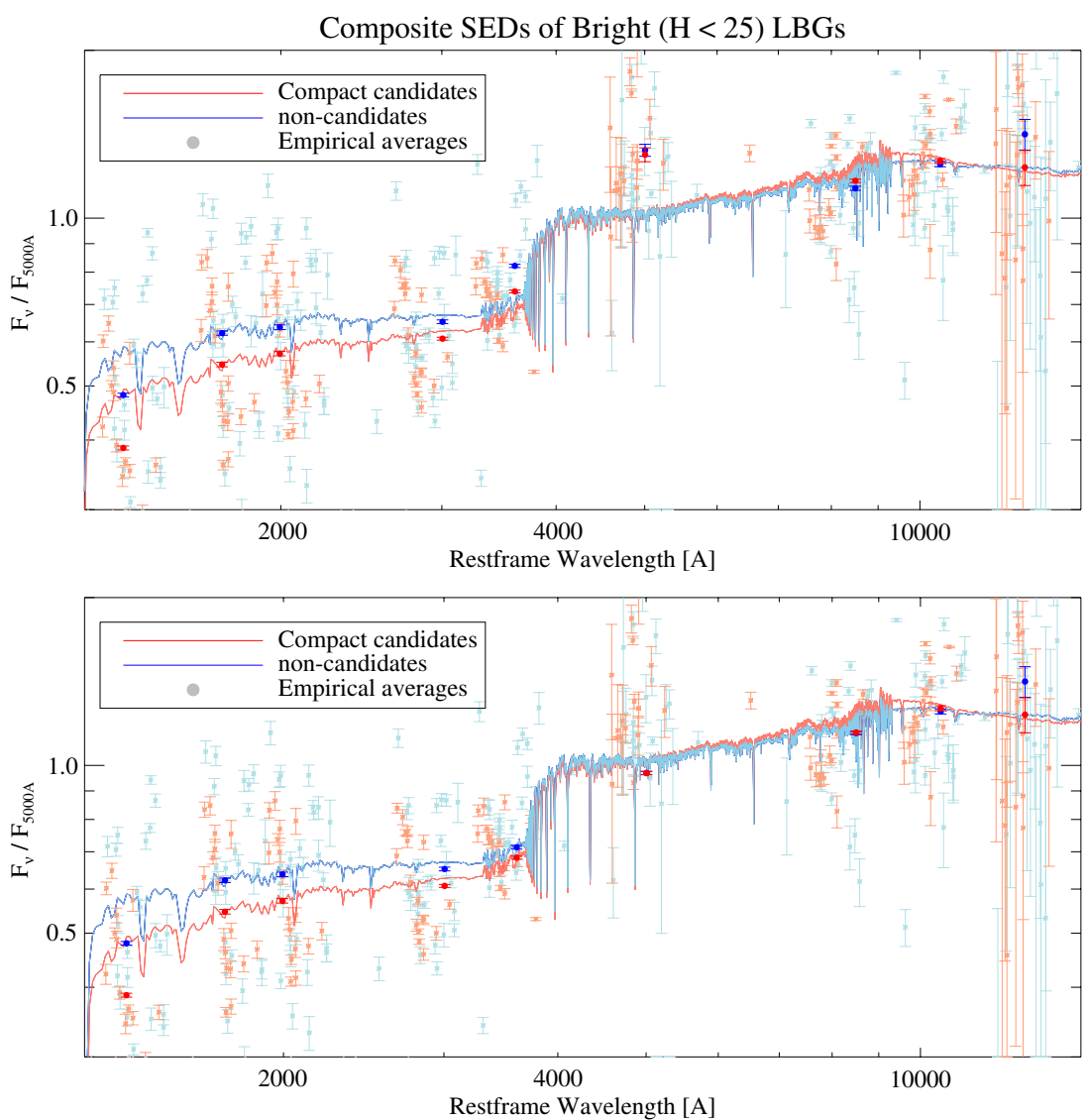

Figure 8. Composite SED templates (lines) and composite of observed SEDs (circles) for the candidates (red) and non-candidates (blue) with spectroscopic redshifts (top panel). Observed photometry for the individual candidates and non-candidates with spectroscopic redshifts are also shown for candidates (salmon) and noncandidates (light blue). Bottom panel: same as the top panel, except photometric points that may be affected by emission lines have been excluded from the composite.

(A color version of this figure is available in the online journal.)

model SEDs are normalized in the same way as the observed data and the averages for candidates and non-candidates are included for comparison. Varying dust does not reproduce well the difference in composite SEDs because of the similarities in the optical part of the SEDs of both candidate and non-candidate samples. Varying age is a better description of the observed trends.

A better look at the photometric differences between candidates and non-candidates and how they may vary with trends in age and dust can be seen in Figure 10, which shows the observed $V-z$ and IRAC channels 1-3 colors of LBGs. This compares colors blue-ward of the 4000 A break with colors red-ward, which are the key features of the differing average SEDs. There is overlap in color distributions of the candidates and non-candidate samples, but the mean of the candidates are offset red-ward in $V-z$ from the non-candidates, but not offset red-ward in IRAC colors. The same color distribution is present among the LBGs that have photometric redshifts (bottom panel), indicating this difference in color persists in the entire LBG sample. Tracks of a sample Bruzual \& Charlot (2003) template SED with varying age and one with varying dust obscuration show that varying age changes $V-z$ color but not IRAC colors, while varying dust changes both colors. This is consistent with, although does not prove, a difference in the average stellar age (since peak of the starburst) between the two samples, with the candidate ETG progenitors appearing older than non-candidates. We note that similar trends are also seen in the $U-V$ versus $V-J$ color-color diagram presented in Figure 7, where candidates tend to be redder in $U-V$, but do not appear redder in $V-J$ color than the non-candidates. Although the dispersion in colors between candidates and non-candidates in Figure 10 overlap, we note that the difference in average SEDs are significant, as determined by the more robustly determined error bars from the simulations presented in Figure 8.

\subsubsection{Infrared Properties}

Here we present further investigation of the potential contribution of dust to the redder restframe-UV SED of the candidates as compared with that of the non-candidates, using the Spitzer/MIPS $24 \mu \mathrm{m}$ (Magnelli et al. 2011; M. Dickinson et al., in preparation) and Herschel/PACS $100 \mu \mathrm{m}$ data (Elbaz et al. 2011; Lutz et al. 2011). The $3 \sigma$ detection limit of the $24 \mu \mathrm{m}$ catalog is $20 \mu \mathrm{Jy}$, and for the $100 \mu \mathrm{m}$ catalog (based on prior information of the $24 \mu \mathrm{m}$ catalog) is $0.8 \mathrm{mJy}$ (Elbaz et al. 2011). In general, the LBGs have a low detection rate $(<8 \%)$ at both $24 \mu \mathrm{m}$ and $100 \mu \mathrm{m}$. Of the candidates, $4 \%$ are within 1 arcsecond of a $24 \mu \mathrm{m}$ detection and $2 \%$ are within 1 arcsecond of a $100 \mu \mathrm{m}$ detection. Of the non-candidates, $8 \%$ are within 1 arcsecond of a $24 \mu \mathrm{m}$ detection and 3\% are within 1 arcsecond of a $100 \mu \mathrm{m}$ detection.

Although the detection rate is low, we checked whether these galaxies should have infrared (IR) fluxes above the detection limits of those surveys, given the amount of dust obscuration 


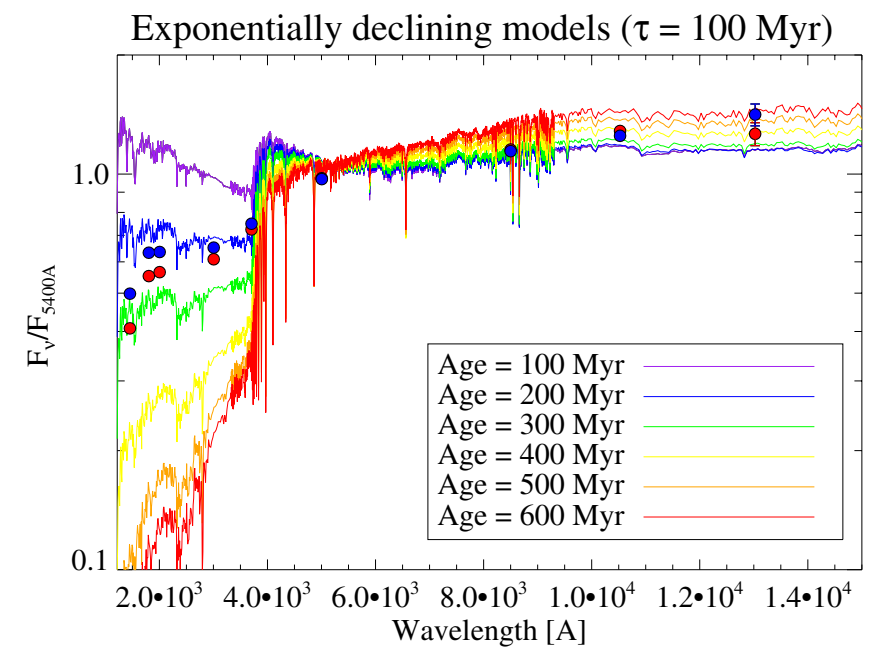

Dust Obscuration (For $\tau=$ age $=100 \mathrm{Myr}$ Model)
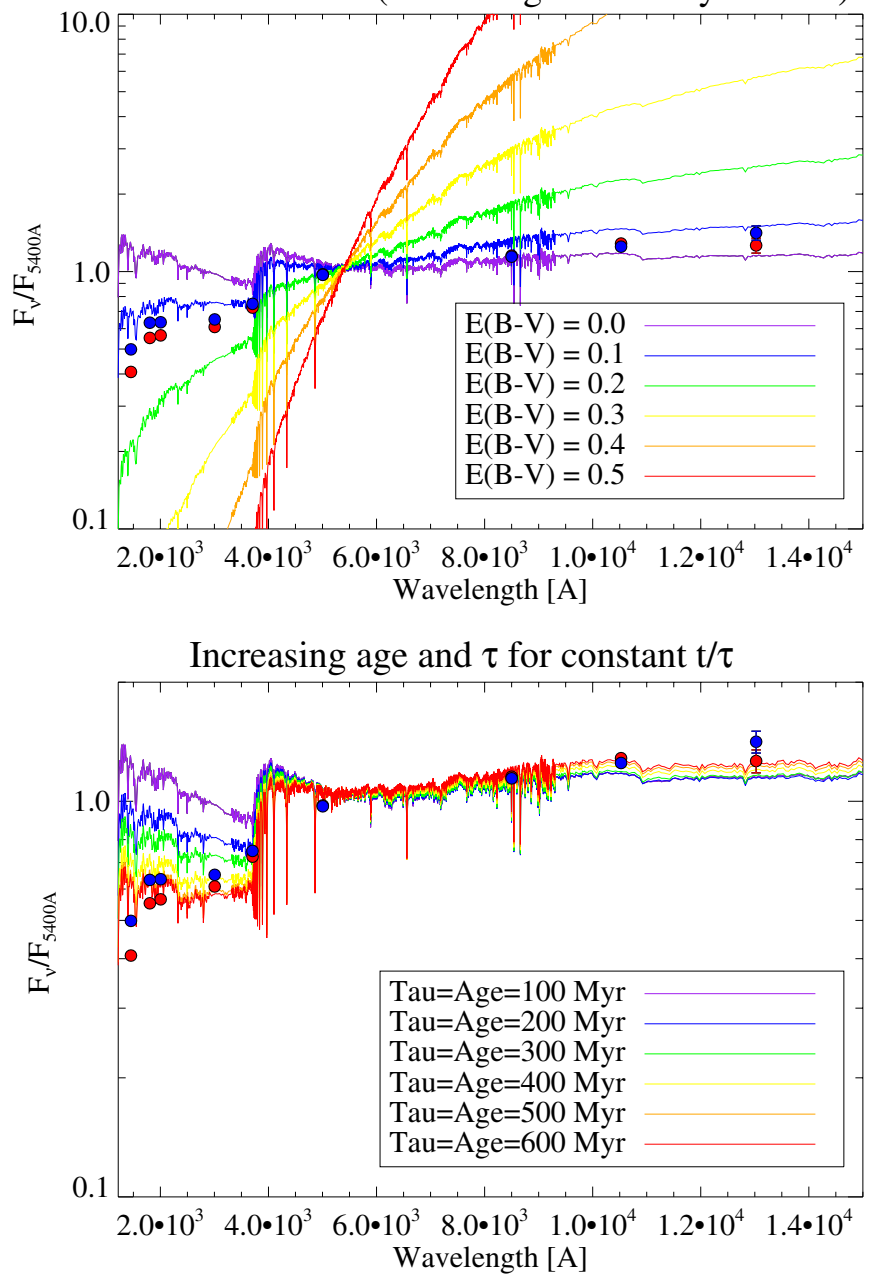

Figure 9. Comparison of normalized SEDs for various exponentially declining SFHs with various age, tau, and dust. Top panel: model with $\tau=100 \mathrm{Myr}$, $E(B-V)=0$, varying age of the burst. Middle panel: model with $\tau=100 \mathrm{Myr}$ and age $=100 \mathrm{Myr}$, varying $E(B-V)$. Bottom panel: model with $E(B-V)=0$, varying both age and $\tau$ such that the ratio remains constant. Composite SEDs of candidates (red) and non-candidates (blue) are included. Varying dust does not reproduce well the variation of the UV while keeping the optical-NIR part flat.

(A color version of this figure is available in the online journal.) that can be inferred from their measured UV-corrected SFRs. To see whether our LBGs should be detected given this estimated amount of dust-obscured SF, we first estimate the IR luminosity $\left(L_{\mathrm{IR}}(\right.$ total $)=L(8-1000 \mu \mathrm{m})$ from the amount of obscured SFR in each galaxy. The obscured SFR is derived from the UVcorrected SFR and the UV-uncorrected (i.e., measured directly from the restframe-UV SED) SFR. To estimate the expected flux densities at 24 and $100 \mu \mathrm{m}$, we use the IR template of Chary \& Elbaz (2001), whose total IR luminosity best matches that estimated for each LBG, convolved with each bandpass. We find that less than $3(2) \%$ of the non-candidates are expected to be detected at 24(100) $\mu \mathrm{m}$, and less than $7(7) \%$ of the candidates would be detected. We also test the inferred flux densities using the updated templates of Elbaz et al. (2011) for main-sequence galaxies and find comparable results. These are generally consistent with our findings listed earlier for the actual number of LBGs with IR counterparts.

Because the majority of these LBGs are below the detection limit of the Spitzer/MIPS and Herschel/PACS surveys, we study the average dust properties with a stacking analysis. Because the detection rate within a one arcsecond search radius is so low, we adopt the following procedure for both wavelengths to carry out the stacking. For objects that are within 1 arcsecond of a detection, we use a $42 \times 42$ pixel image $(50 \times 50$ arcseconds) from the real observed $24 \mu \mathrm{m}$ or $100 \mu \mathrm{m}$ image. For objects that are not detected (more than 90\%), we use a $42 \times 42$ pixel image from the residual map at $24 \mu \mathrm{m}$ or $100 \mu \mathrm{m}$, where the flux from formally detected objects has been removed using the PSF, following the methods of Magnelli et al. (2011) at $24 \mu \mathrm{m}$ and Elbaz et al. (2011) at $100 \mu \mathrm{m}$. The residual map thus includes low-level IR emission from non-detected sources and noise but no flux from neighboring detections. Using the residual map for non-detections minimizes the flux of nearby bright but unrelated IR sources contaminating the LBG stacked flux. We then stack the images of the candidates and non-candidates using a weighted averaging based on the rms maps in the case of the $24 \mu \mathrm{m}$ stack and the weight maps in the case of the $100 \mu \mathrm{m}$ maps. Stacked fluxes are determined by performing aperture photometry on the stacked images and the published aperture corrections from Engelbracht et al. (2007) and the Herschel/ PACS technical documentation. The uncertainty on the stacked fluxes for candidates and non-candidates is determined by the following procedure. We repeat the aforementioned stacking for the same number of random positions in the maps as galaxies in the candidate and non-candidate samples. We generate 1000 sets of these random stacks and use the standard deviation of the stacked fluxes of these random positions as the uncertainties of each sample's stacked flux.

The results from the stacking analysis are shown in Figure 11. We find that candidates and non-candidates have statistically indistinguishable and non-significant stacked flux at $100 \mu \mathrm{m}$. For candidates, we find $F(100 \mu \mathrm{m})_{\text {stack }}=50 \pm 169 \mu \mathrm{Jy}$ and for non-candidates, we find $F(100 \mu \mathrm{m})_{\text {stack }}=-15 \pm$ $87 \mu \mathrm{Jy}$. The candidates have no significant stacked emission at $24 \mu \mathrm{m}$, while the non-candidates do have some significant stacked flux. For candidates, we find $F(24 \mu \mathrm{m})_{\text {stack }}=3 \pm 3 \mu \mathrm{Jy}$ and for non-candidates, we find $F(24 \mu \mathrm{m})_{\text {stack }}=9 \pm 2 \mu \mathrm{Jy}$, an approximately $4 \sigma$ detection. This supports our hypothesis that the candidates are redder because of older ages rather than dust because the candidates do not show evidence of higher dust emission, and the non-candidates appear to have more dust emission than the candidates on average. 

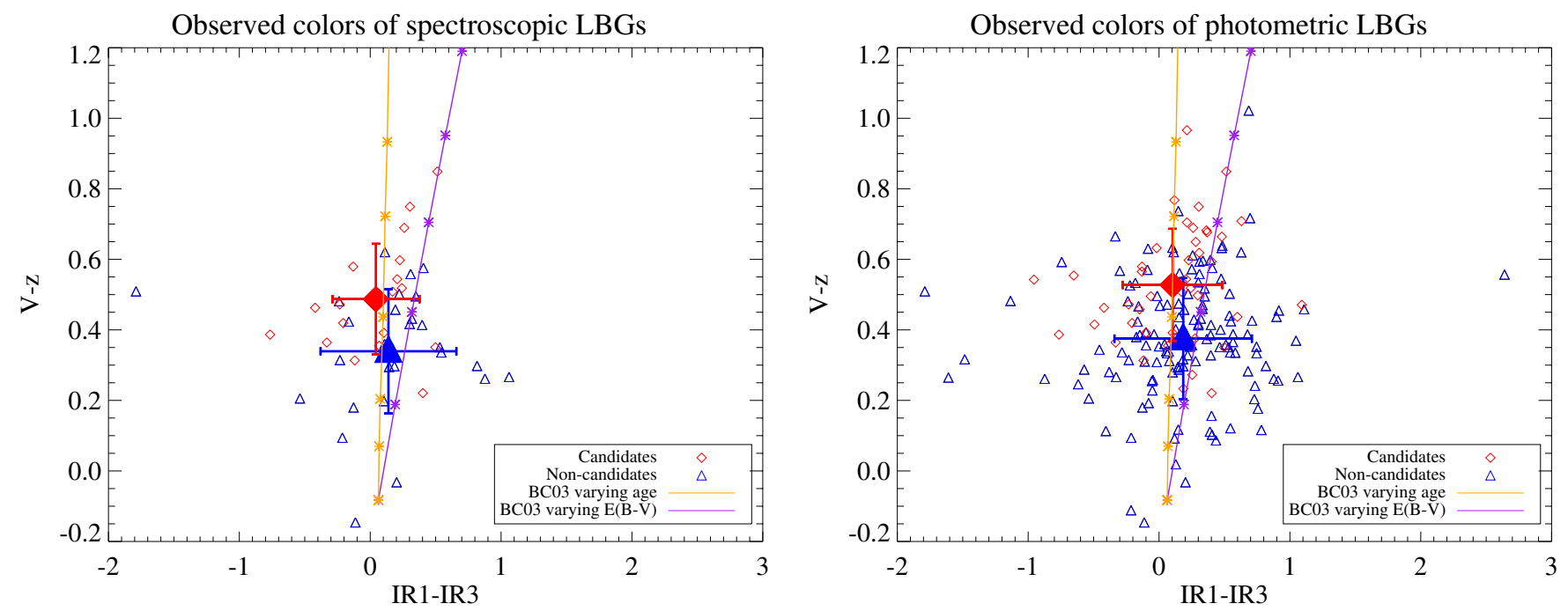

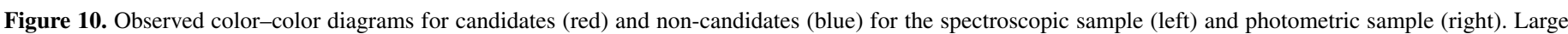

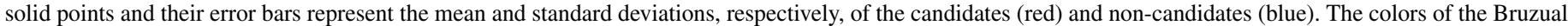

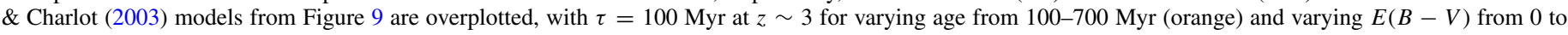
0.7 (purple).

(A color version of this figure is available in the online journal.)

\subsubsection{X-Ray Stacking}

Of particular importance to studies of star-forming and compact galaxies, a class to which our candidate LBG sample belongs, is the contribution of an active galactic nucleus (AGN). Some galaxies whose flux is dominated by that of an AGN may, in effect, appear compact in terms of bolometric output, but are not compact in terms of their stellar density. Rather, they may simply be out-shined by the AGN. X-ray detections amongst our two LBG samples thus play an important role in understanding the contribution (if any) to contamination in our sample from bolometrically dominating AGN.

To assess the fraction of AGN present among the candidate sample relative to the $\mathrm{LBG}$ sample as a whole, we have matched the LBG samples to X-ray detections from the $4 \mathrm{Ms}$ observations with the Chandra X-Ray Observatory (Xue et al. 2011). The overwhelming majority of the LBGs are not detected in the 4 Ms observations, with the exception of three sources from the non-candidate sample. Similarly low detection rates for LBGs have been found by Hathi et al. (2013). Nevertheless, since the non-compact sample is larger than the compact one, we cannot infer anything about the X-ray emission frequency in the two samples. We further assess differences in X-ray properties between the two samples using stacking of the $4 \mathrm{Ms}$ imaging. In both the candidate and non-candidate samples, the X-ray stack showed no statistically significant X-ray emission, with an upper limit on X-ray luminosity of $10^{43} \mathrm{erg} \mathrm{s}^{-1}$. This result suggests that our candidate sample is not contaminated by AGN any more than the non-candidates.

\subsection{The UV and Optical Morphology and Size of The LBG Samples}

In this section, we study the morphology and size of the young and old stellar populations of the galaxies in each sample. The aims of this analysis are (1) to explore faint surface-brightness features (e.g., Hathi et al. 2008a) and (2) to study whether or not there are morphological differences in the two samples that can help us assess whether their merging histories differ.
Because our objects are faint $(H<25)$ and because we are interested in low surface-brightness features that may be too faint to observe on an individual galaxy basis, we study the average distribution of the stellar populations by stacking the two samples. We stack the $H S T /$ WFC3 $H$ band, which is in rest-frame optical at $z \sim 3$ and therefore probes the older stellar population. We also stack the HST / ACS ( $z$ band) images, which are at the rest-frame UV and therefore probe the young stars and star-forming regions. To produce the stacked images, we generate images in each band in which we have masked out any neighboring galaxy that is not also selected as a $U$-band dropout by our color selection. To mask the emission from these interlopers, we use the corresponding segmentation map from the SExtractor detection process in each band. The images are then shifted so that each image is centered at the position of the peak of the $H$-band emission. To perform the stack, we do an inverse-variance weighted mean of each pixel, using the map rms plus Poisson noise to compute the weights. This weighting scheme ensures that possible non-azimuthally symmetric lowsurface brightness structures will be preserved in the final stack. We then measure average structural properties of the galaxies using GALFIT and make azimuthally averaged light profiles using the IRAF function ellipse.

As mentioned in Section 4.1, given their redshift distribution, many of the LBGs may have optical emission lines entering the $H$ band (primarily the [O II] $\lambda 3727$, but also $\mathrm{H} \beta$ ), and this may bias the $H$-band light distribution towards the starforming regions and therefore would not trace the older stars. The $J$ band is largely unaffected by these emission lines. We therefore compare the $H$-band stacks and $J$-band stacks to check for discrepancies, which may indicate contamination from this line emission. The light profiles for the $H$ band and $J$ band, for candidates and non-candidates, are shown in Figure 12 . This figure shows that the $H$-band and $J$-band stacks, on average, have very similar profiles for each sample, showing that any contamination to the $H$ band from emission lines is negligible.

In Figure 13, we compare the average, peak-flux normalized $H$-band light profiles for the candidates and non-candidates. 

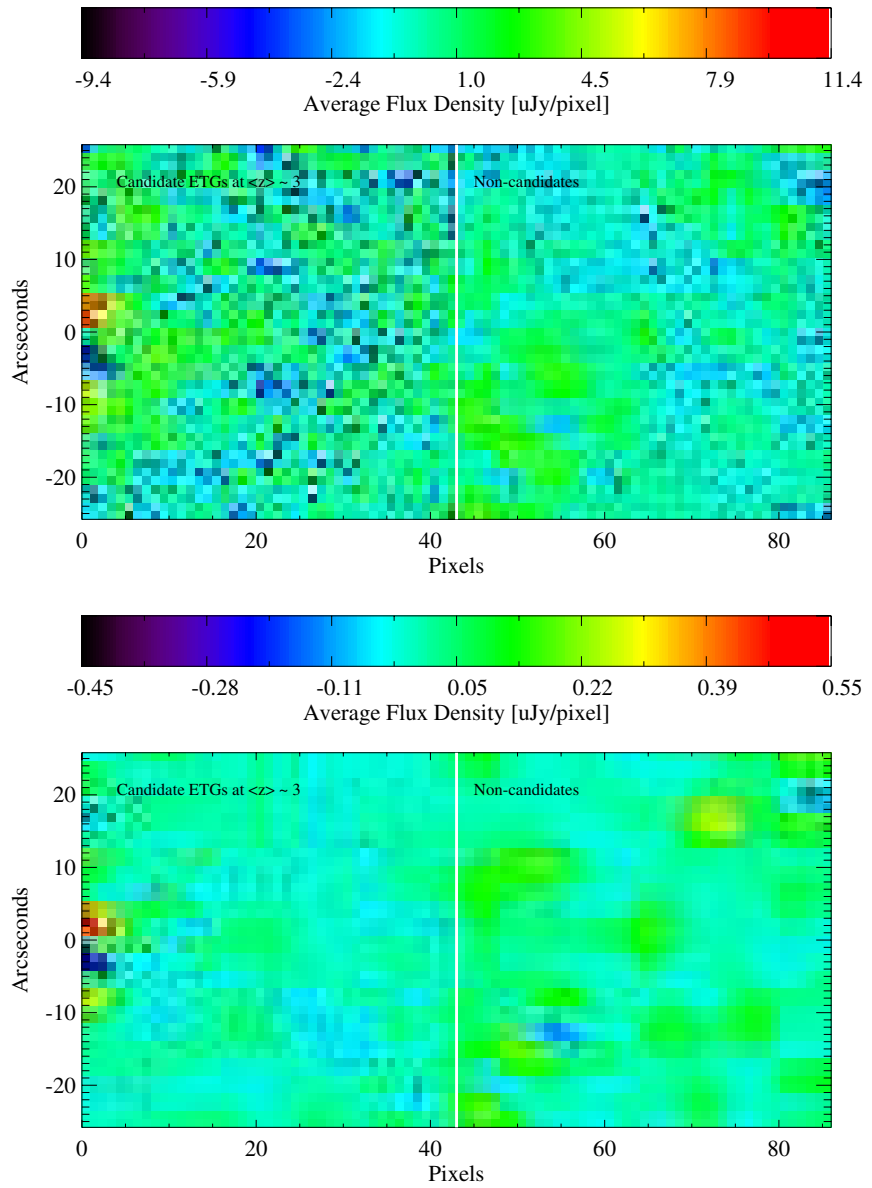

Figure 11. Top panel: stacked Herschel/PACS $100 \mu \mathrm{m}$ image at the positions of the candidate LBGs (left) and non-candidates (right). No significant stacked emission is present in either sample. Bottom panel: stacked Spitzer/MIPS $24 \mu \mathrm{m}$ image at the positions of the candidate LBGs (left) and non-candidates (right).

(A color version of this figure is available in the online journal.)

We also repeat the stacking using the Cassata et al. (2013) compact ETG sample between $1.2<z<2.8$, where we mask out all neighboring galaxies prior to stacking. This comparison between compact candidates (red) and non-candidates (blue) in this figure essentially reflects our selection criteria: candidates must be compact (thus have smaller radii) and tend to be more star-forming, resulting in a higher peak surface brightness than non-candidates. (Because of the flux normalization in Figure 13, the peaks are coincident, but this fact can be seen from the absolute difference between peak and normalized noise level and is also visible comparing the unnormalized peak flux for candidates and non-candidates in the two panels of Figure 12). We include in Figure 13 the stacked average light profile of ultra-compact, compact, and non-compact ETGs as defined in Section 3 from the Cassata et al. (2013) sample, which compares the peak-flux-normalized shape of the light profiles. The figure shows that not only do the ultra-compact ETG sample and our LBG candidate sample have, on average, the same half-light radius (because of the way they have been selected), but also nearly identically steep light profile overall. This presentation with the peak-flux normalization highlights the actual similarity in steepness of the light profiles. It is important to note here that simply comparing Sersic parameters does not adequately highlight the similarity because of the covariance of half-light radius and Sersic index. Last, we note that the average light
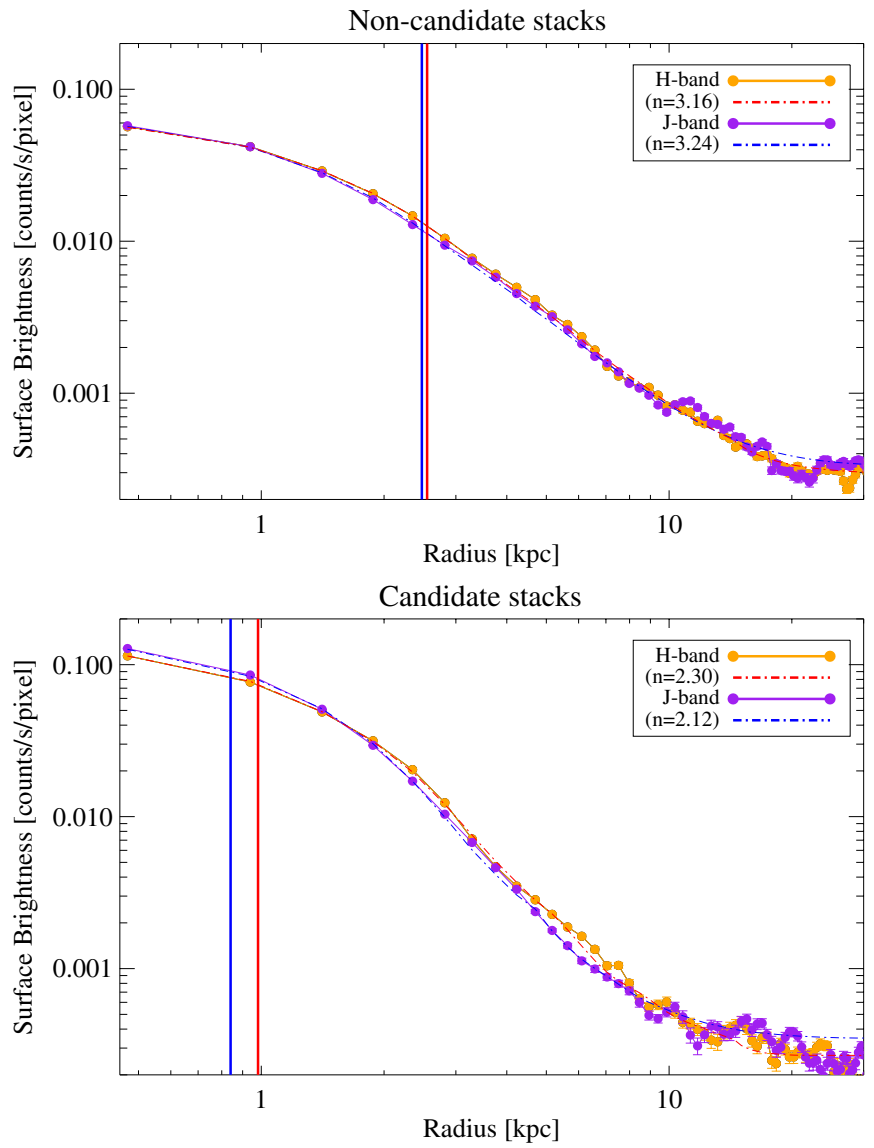

Figure 12. $J$-band and $H$-band stacked light profiles for non-candidates (top) and candidates (bottom). Points connected with solid lines indicate observed light profiles, and dot-dashed lines are the best-fit Sersic profiles as measured by GALFIT. Vertical lines indicate effective radius of each GALFIT model. In general, the structural properties in the two bands are very similar, suggesting that if emission lines from SF regions are entering the $H$-band bandpass, it is not significantly biasing our measure of the distribution of the old stellar populations.

(A color version of this figure is available in the online journal.)

profile of all the samples considered here shows no evidence of excess flux above a Sersic profile, a signature that could imply the presence of tidal debris from recent merging.

As mentioned previously, of particular importance to understanding the mechanism by which these galaxies build up their stellar mass over time is the relative spatial distribution of young and old stars. In Figure 14, we compare the average stacked light profiles of the restframe UV ( $z$ band) with the restframe optical ( $H$ band) for our candidates and non-candidates. In this figure, the differing PSFs have been fully taken into account by GALFIT when estimating the Sersic parameters, and are plotted after convolution with their respective PSFs. In both candidates and non-candidates, it is clear that the UV flux is more centrally concentrated than the optical flux, as indicated by the fact that the UV half-light radius of each is smaller than that of the optical, lending support to our assumption in Section 3 that these galaxies gain stellar mass through centralized, in situ star formation, rather than in the outskirts for example, and therefore may build the stellar masses of galaxies at constant (or modestly increasing) size, consistent with the evolutionary path we assume in Figure 3 (for a similar discussion on how galaxies grow in mass and size see also Ownsworth et al. (2012), who do not consider the case of compact galaxies as we do here). 
Table 1

Volume Densities of High-redshift Galaxies

\begin{tabular}{lccc}
\hline \hline Galaxy Type & Notes & Co-Moving Volume Density & Reference \\
\hline $\begin{array}{l}\text { Submillimeter Galaxies } \\
\text { Submillimeter Galaxies }\end{array}$ & $\begin{array}{c}\text { As observed } \\
\text { Incl. duty cycle }\end{array}$ & $1 \times 10^{-5}$ & e.g., Scott et al. (2002) \\
& & $1-3 \times 10^{-4}$ & $\begin{array}{c}\text { Chapman et al. (2005), } \\
\text { Swinbank et al. (2006) } \\
\text { e.g., Magnelli et al. (2011) } \\
\text { ULIRGs }\end{array}$ \\
Ultra-compact ETGs & As observed & $7.5 \times 10^{-5}$ & Cassata et al. (2013) \\
Compact ETGs & $1.2<z<2.8$ & $7.4( \pm 1.1) \times 10^{-5}$ & Cassata et al. (2013) \\
Non-compact ETGs & $1.2<z<2.8$ & $1.3( \pm 0.1) \times 10^{-4}$ & Cassata et al. (2013) \\
Compact candidate LBGs & $1.2<z<2.8$ & $5.5( \pm 0.9) \times 10^{-5}$ & This study \\
Non-candidate LBGs & $3<z<4$ & $1.2( \pm 0.2) \times 10^{-4}$ & This study \\
All $H<25$ LBGs & $3<z<4$ & $3.7( \pm 0.3) \times 10^{-4}$ & This study
\end{tabular}

Note. ${ }^{\text {a }}$ In units of $\mathrm{Mpc}^{-3}$.

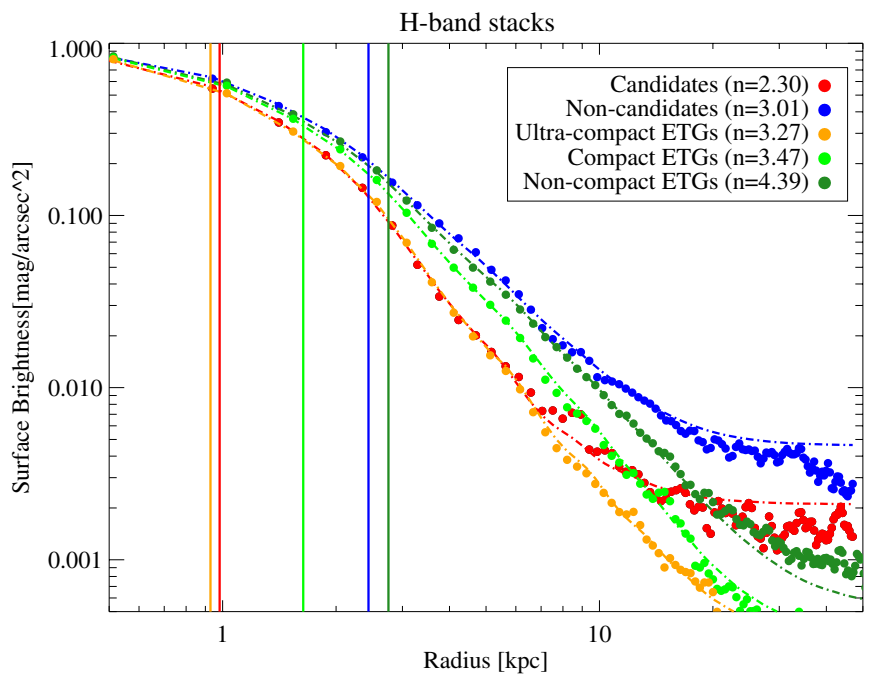

Figure 13. Normalized light profiles (points) from the $H$-band stacks of candidates (red), non-candidates (blue) along with the those of the three classes of ETG samples of Cassata et al. (2013). The three compactness classes are as defined in Section 3: non-compact (dark green), compact (green), and ultracompact (orange). Best-fitting Sersic profiles to the stacks measured by GALFIT are dashed lines, and measured half-light radius represented by vertical lines, and Sersic index as indicated in the legend.

(A color version of this figure is available in the online journal.)

\subsection{Number Densities of Candidates and Real ETGs}

If major merging has not been a driving mechanism, then the co-moving number densities of our candidate progenitors and those of the compact ETGs should be comparable. In the literature, co-moving number densities have been used as arguments favoring massive dusty starbursts, such as ultraluminous IR galaxies (ULIRGs) and submillimeter galaxies (SMGs), as being the progenitors of local massive ellipticals in the centers of galaxy clusters (Swinbank et al. 2006; Daddi et al. 2009). These galaxies are relatively rare $\left(n \sim 10^{-5} \mathrm{Mpc}^{-3}\right.$; Scott et al. 2002; Chapman et al. 2005) like the high-redshift ETGs $\left(n \sim 5.5( \pm 0.8) \times 10^{-5}\right.$ for non-compact ETGs and $n \sim 1.3( \pm 0.1) \times 10^{-4}$ for compact ETGs; Cassata et al. 2013). See Table 1 for a comparison of number densities. Because of their extreme nature, SMGs and ULIRGs may be capable of forming the requisite stellar mass at high redshift (Lilly et al. 1999), as well as having sufficient gas content to produce compact remnants (Tacconi et al. 2008, 2006). (We will discuss the similarities between SMGs and compact ETGs further in Section 5.2.) Evolutionary scenarios for SMGs can in principle
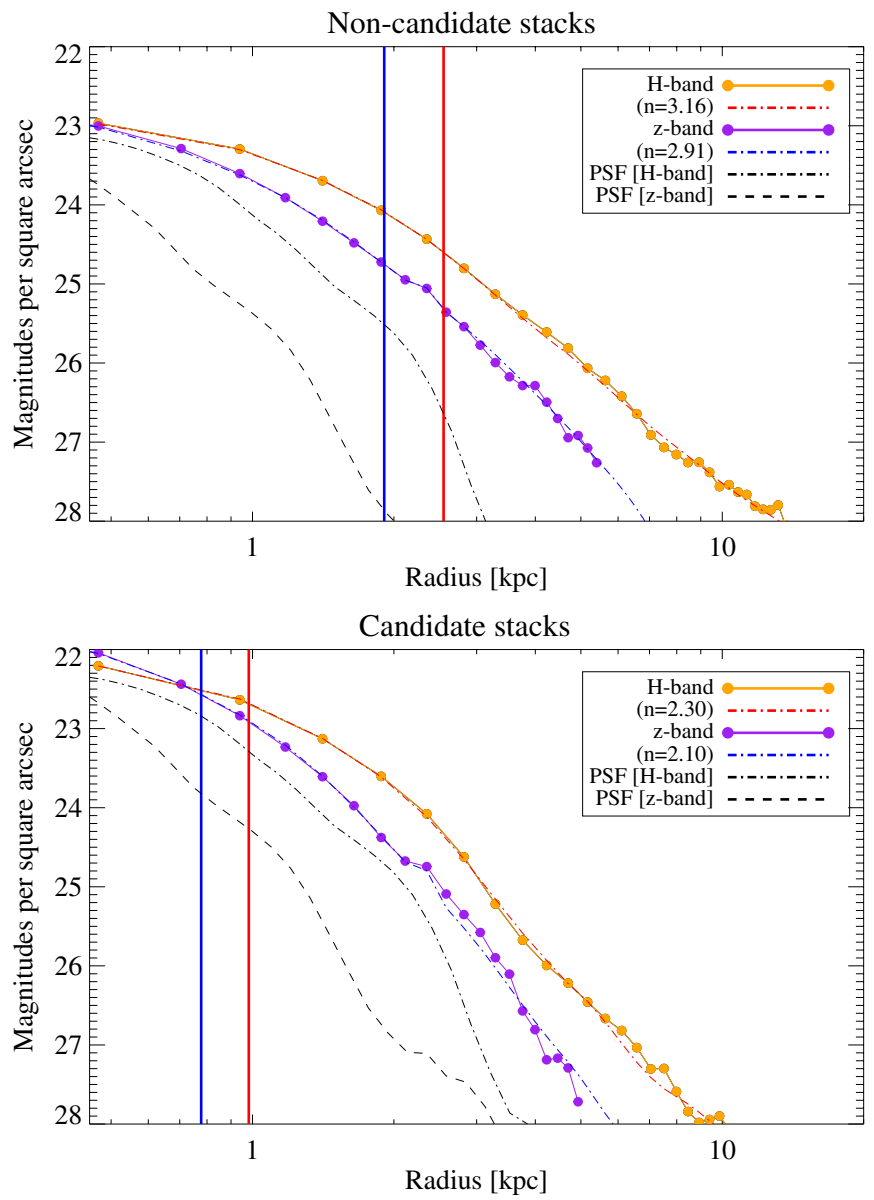

Figure 14. $z$-band and $H$-band stacked light profiles for non-candidates (top) and candidates (bottom). Points connected with solid lines indicate observed light profiles and dot-dashed lines are the best-fit Sersic profiles as measured by GALFIT, convolved with the appropriate PSF in each band. Vertical lines indicate effective radius of each GALFIT model.

(A color version of this figure is available in the online journal.)

be tested using galaxy clustering (e.g., Hickox et al. 2012); however, current samples of high-redshift compact ETGs is prohibitively small for this measure and comparison. Larger samples using the full CANDELS survey data may contain enough compact ETGs for this purpose in the future.

For now, co-moving number densities provide a first order consistency check. It is important to keep in mind, however, that an accurate comparison of the spatial abundances of the 
two populations would require not just quantifying the effective amount of major merging that takes place both before and after the star-forming galaxies quench, but also the fact the LBG selection does not recover all the star-forming galaxies at $z \sim 3$ (e.g., Guo et al. 2012; Marchesini et al. 2010; Muzzin et al. 2013). In any case, if our sample of compact candidates truly contains the progenitors of compact ETGs, of which the majority grow their stellar mass in situ (i.e., steady growth independent of merging) and evolve into compact ETGs by quenching, then the co-moving number densities of compact candidate LBGs must at least be large enough to account for the observed number densities of compact ETGs. We measure the co-moving number density of our LBG samples by directly integrating the redshift distribution to get the cosmological volume sampled by the galaxies:

$$
\text { Volume }=\frac{\int N(z) \frac{d V(z)}{d z} d z}{\int N(z) d z}
$$

where $N(z)$ is the redshift distribution of each LBG sample. We then use this to get the observed number density of LBGs in each sample:

$$
n=\frac{N_{\text {tot }}}{\text { Volume }}
$$

where $N_{\text {tot }}$ is the number of LBGs in each sample. The co-moving number density of the Cassata et al. (2013) compact ETGs (stellar density $>3 \times 10^{9} M_{\odot} \mathrm{kpc}^{-2}$ ) is $1.3( \pm 0.1) \times$ $10^{-4} \mathrm{Mpc}^{-3}$ between $1.2<z<2.8$. Co-moving number densities of other samples of compact ETGs from other samples have similar values within this redshift and mass range (Barro et al. 2013; Patel et al. 2013).

We find that our candidate compact ETG progenitors have a co-moving volume density of $1.2 \times 10^{-4} \pm 0.2 \mathrm{Mpc}^{-3}$, consistent within the uncertainty with the volume density of the Cassata et al. (2013) sample. More specifically, the compact candidate sample can account for $\sim 92 \%$ of the compact ETGs by number density found at lower redshifts. Although within the uncertainty this can account for all detected compact ETGs, this does not account for the destruction of compact galaxies through merging or rejuvenation of star formation between $z \sim 3$ and $z \sim 1$ 1.6. In Section 4.4, we will discuss a fraction of compact $z \sim 3$ galaxies that we find are missed by our LBG selection criteria and how this fraction affects the aforementioned estimate of number density.

\subsection{Compact Progenitors Missed by the LBG Color Selection}

We have chosen to use the LBG color selection for our sample in the aforementioned analysis because of its efficiency, lack of interloper contamination (with $U$-band dropouts the only modest source of contamination is that by galactic stars, which are easily identified in the Hubble Space Telescope (HST) images and removed), and the fact that it is largely model independent (see Giavalisco 2002). It also avoids the potential bias caused by the degeneracy between dust obscuration and age, which could result in including galaxies with a low specific SFR among the progenitors.

In any case, a more general search for candidate progenitors can be done using a sample selection on the basis of photometric redshifts and subsequent SED fitting to spectral libraries to derive stellar mass. In fact, a fraction of $z \sim 3$ galaxies with spectroscopic redshifts are missed by our color selection, primarily because they reside in crowded fields where their TFIT photometry may be affected by nearby $U$-band detected galaxies, causing them to be excluded from our sample of $U$-band dropouts. Therefore, we briefly present again here our main results for candidates and non-candidates selected in an identical way as the LBG candidates and non-candidates, but this time from all galaxies with photometric or spectroscopic redshifts between $3<z<4$. We will call this sample our SED-selected sample.

In general, the SED-selected samples are larger, with the number of non-candidates increasing by $45 \%$ and the number of candidates almost doubling, with an increase of $95 \%$. Not surprisingly, there is some significant overlap between the LBGand SED-selected samples. Of the 136 LBG-selected noncandidates, 113 are also SED-selected non-candidates. Of the 44 LBG-selected candidates, 42 of them are also SED-selected candidates. (The two LBGs not present in the SED sample are galaxies with photometric redshifts between $2<z<3$ discussed in Section 3). We stress these are not independent samples, and therefore this is not an independent test of our results. Rather, adding the results from the SED-selected sample serves to augment our analysis with an increased sample size that is not biased by the fact that the LBG sample selects galaxies on the basis of their UV emission, and therefore cannot be too dusty. We also note that while the LBG samples studied in Section 4 have similar $24 \mu \mathrm{m}$ detection rates $(<8 \%)$, the SEDselected non-candidates remain with a low-IR detection $(6 \%)$ and the SED-selected candidates jump to a $20 \%$ IR detection rate.

In Figure 15 we present the same analysis of average SEDs presented in Section 4.1, this time using the SED-selected candidate and non-candidate samples. The top panel shows that candidates are redder, in both the restframe UV and the optical parts of the spectrum, suggesting a dustier average SED. This is no doubt reflecting the increased fraction of IR detections among the candidates using the SED selection. However, because the number of IR detections in each sample is still a small fraction of the total, we have repeated the analysis without including the IR-detected galaxies. The bottom panel shows this comparison of non-IR-detected candidates and non-candidates, and shows the same signature of older stellar ages that was seen for the LBG sample in Figure 8. This figure, combined with the results of Section 4 , is evidence that $3<z<4$ galaxies that are selected to be compact in stellar density have, on average, a redder SED that is best explained by an aging stellar burst. This supports our key result found for the LBGs in Section 4, namely that there is a correlation between the compactness of galaxies and the average age of their forming stellar populations, the sense being that more compact galaxies have older forming populations, i.e., the starburst is older.

This SED-selected sample of compact candidates has a larger co-moving number density of $2.6 \times 10^{-4} \pm 0.3 \mathrm{Mpc}^{-3}$ compared with $1.2 \times 10^{-4} \mathrm{Mpc}^{-3}$ for the LBG-selected compact candidates. This number density of SED-selected compact candidates (which includes $95 \%$ of the LBG candidates) is a factor of $\sim$ two larger than the compact ETGs.

\section{DISCUSSION}

The key point of this paper is the identification of physically motivated candidate progenitors of the massive, compact ETGs observed at $z \gtrsim 1.2$. Both individual images and very deep stacks show that the light profile of the massive compact ETGs does not have any diffuse light in excess of their extremely 

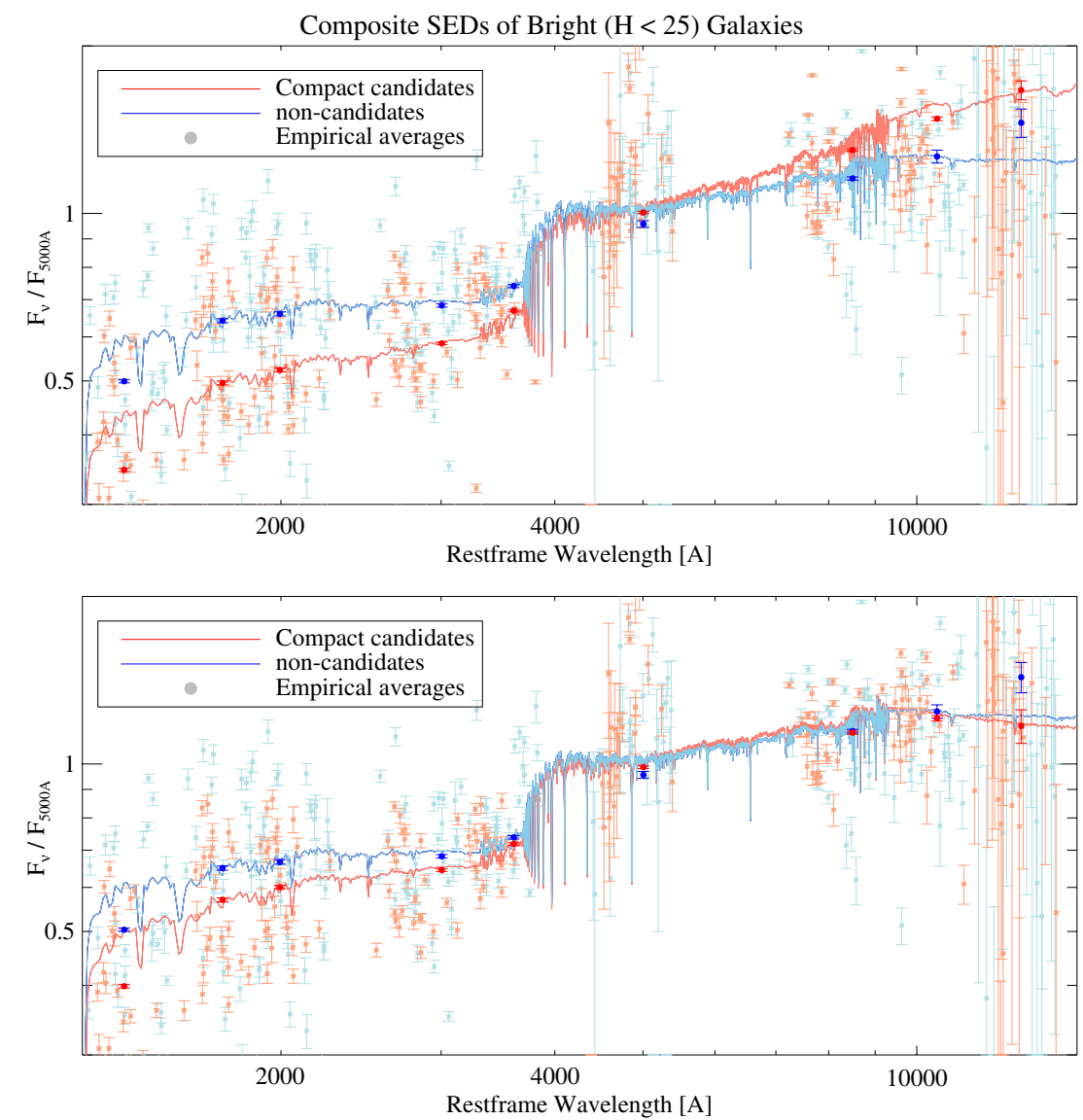

Figure 15. Average SEDs, as calculated in Figure 8, for candidates and non-candidates selected on the basis of spectroscopic redshifts rather than LBG color selection. Top panel: all candidates and non-candidates. Bottom panel: the same sample with $24 \mu \mathrm{m}$ detected galaxies removed. Removing the small fraction of IR-detected galaxies shows the same result as that from the LBG selection, indicating older ages among the candidates.

(A color version of this figure is available in the online journal.)

compact cores, approximated by a steep Sersic profile with typical parameters $n=3.6$ and $r_{e}=1.2 \mathrm{kpc}$. Simulations show that merging events rearrange the stellar mass profile of the merging partners in a way that the profile of the merger remnant is more diffuse than that of the initial partners (e.g., Lotz et al. 2010; Wuyts et al. 2010). Dissipative processes in a wet merger might channel gas to a nuclear region and produce a massive, compact component in a starburst episode; however, the preexisting diffuse component would remain visible and none is observed. This implies that star-forming progenitors of the compact ETGs must be at least as compact themselves, and thus we hypothesized that their progenitors may be found among compact star-forming galaxies at higher redshifts.

We looked for such progenitors and found some plausible candidates among LBGs at $z \sim 3$. The candidate progenitors have been chosen to have similar size and morphology to the ETGs at $z \gtrsim 1.2$, and mass and SFRs such that after they quench their star-formation activity and would be classified as "passive", e.g., according the definition given by Cassata et al. $(2011,2013)$, their stellar mass and projected stellar densities are in line with the analogous properties of the ETGs. It is important to understand that the selection of the candidate progenitors depends only mildly on the details of the assumed time evolution of the quenching phase of the LBGs. Much more important parameters in determining whether a given LBG is a candidate progenitor or not are its physical size, its SFR, and, to a lesser extent, its stellar mass at the time of observation, i.e., at $z \gtrsim 3$. All of the observed $z \gtrsim 1.2$ massive, passive galaxies with stellar mass $M>10^{10} M_{\odot}$ can be accounted for with progenitors selected from LBG; we found that a factor of two more candidate progenitors are found if more general selection criteria for star-forming galaxies are adopted. With the addition of these new candidates, the evolutionary constraints between these two populations is relaxed somewhat; up to half of them may merge and increase size, rejuvenate their star formation, or fail to quench, and there are still sufficient compact candidates to account for the formation of compact ETGs. The recent study by Stefanon et al. (2013), following a similar methodology to that presented here, found relatively few galaxies (five) that may be considered progenitors of compact ETGs (although with higher mass limits of $M>10^{11} M_{\odot}$ for ETGs, and initial $z>3$ star-forming samples with $10^{10.6}>$ $M>10^{11}$ ). Their conclusion for these more massive samples differs from ours in that a significant fraction of progenitors of these more massive compact ETGs must be created between $2<z<3$ (Stefanon et al. suggest through merging). Their sample is considered compact according to our criteria, and therefore likely overlaps with our sample on the massive end. We note that we do find a similar number (nine) of SED-selected massive $\left(M>10^{10.6} M_{\odot}\right)$ galaxies that are already compact at $z>3$. To quantify any differences in the buildup of high-redshift ETGs as a function of stellar mass, for example if merging must contribute to the massive end as suggested by Stefanon et al. (2013), relative to the more general ETG samples studied here where merging is not required, it will be necessary to 
study larger samples, including other CANDELS fields, in the future.

We subsequently studied the properties of the candidate progenitors and compared them to those of the non-candidate LBGs. The most remarkable difference is that the candidate progenitors have significantly redder rest-frame farUV colors than the non-candidates, but essentially identical optical SED. The mid-IR properties of both types of galaxies show that larger dust obscuration of the candidates versus the non-candidates is unlikely to be responsible for the difference. If anything, the non-candidates have larger dust luminosity given their activity of star formation. This forces us to conclude that the redder UV is explained by an older burst (or period) of star formation, namely the phase of star formation of the candidates has progressed more toward quenching it. This is consistent with the general finding that a compact stellar morphology is the best predictor of passivity, i.e., early star-formation quenching, among massive galaxies at $z>1$ (e.g., Bell et al. 2012).

The fact that position of the candidate progenitors on the main sequence is higher than that of the non-candidates is particularly interesting in the context of the discussion of the main sequence of galaxies presented in Renzini (2009). The arguments presented there suggest that galaxies on the main sequence with above-average SFR must quench rapidly and early, resulting in a generally shorter lifetime of star formation. It is therefore interesting that not only do we find that our candidate sample follows such an elevated distribution of SFRs on the main sequence relative to the non-candidates, as seen in Figure 6, but we also find that the candidates that appear to show signs that their star formation will shut down. Qualitatively speaking, the candidates are consistent with this scenario outlined in Renzini (2009) for a more rapid evolution for galaxies that follow the main sequence with higher SFRs.

The rest-frame UV and optical morphology of both the ETGs and of the LBG candidate progenitors show the lack of any diffuse component in excess of the very compact main body of the galaxies. This is observed both in individual galaxies and in deep stacks. In addition, LBG candidate progenitors and ultracompact ETGs show similarly steep profiles (see Figure 13). The fact that star formation is observed to take place only in very compact regions in the LBG progenitor candidates and that no "halo" stars (down to a limit of $\sim 29 \mathrm{mag} \operatorname{arcsec}^{-2}$ ), i.e., no stellar component in excess of the steep light profile of the compact body of the galaxies, are observed in the passive ones is consistent with our suggestion that the growth of stellar mass in these galaxies takes place at essentially constant size, i.e., the stellar density increases with the stellar mass. In turn, this indicates that star formation takes place in these systems via a highly dissipative accretion of gas. We speculate in the following sections that this might be consistent with some ideas on how cold accretion proceeds in massive galaxies (e.g., Dekel et al. 2009a, 2009b; Oser et al. 2010; Sales et al. 2012; Johansson et al. 2012).

\subsection{In Situ Star Formation from Accretionary Mechanisms}

The existence and the spatial abundance of compact candidate progenitors shows that there are sufficient ordinary star-forming galaxies with small size and large stellar mass and SFRs that can evolve through in situ star formation to form compact ETGs without having to invoke special mechanisms that, in a relatively short timescale, can both quench a star-forming galaxy and change its morphology into a compact one, e.g., gas-rich mergers with differential dust obscuration to hide the extended halos (see Wuyts et al. 2010), to explain the emergence of compact ETGs. Rather, simple secular evolution through in situ star formation is sufficient, provided a quenching mechanism exists that can sufficiently remove the cold gas supply rapidly from these galaxies. We discuss various arguments in favor of this scenario below.

\subsection{Comparison to Ultra-luminous IR Galaxies and Submillimeter Galaxies}

ULIRGs and SMGs are often identified as the progenitors of local ETGs, as well as the compact ETGs at high redshift, based on number densities (Daddi et al. 2009; Swinbank et al. 2006), velocity-size relations (Bouché et al. 2007), consistency with the Faber-Jackson relation (Faber \& Jackson 1976) for local ellipticals (Swinbank et al. 2006), and clustering (Blain et al. 2004; Brodwin et al. 2008; Hickox et al. 2012; although see Williams et al. 2011a for the limitations of SMG clustering measurements). The connection between these IR-luminous galaxies and ETGs is based primarily on the high SFRs and stellar masses that are typical of ULIRGs and SMGs, which are capable of producing the requisite stellar mass of compact ETGs on short timescales. Since these galaxies are commonly believed to be the result of major mergers, the quenching of star formation and morphological transformations can be easily tied to the merger. However, studying the stellar distributions of these galaxies is difficult because of their large dust obscurations. This means only small numbers of SMGs have been mapped with the HST at near-IR wavelengths to measure the spatial extent of the bulk of the stellar mass. The results from nearIR mapping indicates the morphologies of SMGs are rather heterogeneous, including SMGs that are large and irregular with multiple components (Smail et al. 2004; Swinbank et al. 2006; Tacconi et al. 2008); dominant components that are disk-like (Targett et al. 2011, 2013); and are, on average, larger in size than compact ETGs (Swinbank et al. 2010; Mosleh et al. 2011; Targett et al. 2011, 2013; Bussmann et al. 2012). Similar results have been found for ULIRGs (Kartaltepe et al. 2012). While a large fraction of these objects appear to be gas-rich mergers (Tacconi et al. 2008; Kartaltepe et al. 2012), collectively these studies suggest SMGs and ULIRGs display a large variety of morphologies and sizes, and as a population are not similar in morphology to compact ETGs. While the SMG or ULIRG merger phase is likely a plausible avenue for quenching massive galaxies (e.g., Hopkins et al. 2006), its unclear how the average SMG can decrease in half-light radius by a factor of two or more after the star formation has been quenched in order to form a compact ETG with the average properties of the Cassata et al. $(2011,2013)$ samples.

\subsection{Formation of Compact Star-forming Galaxies}

The more ordinary LBGs are generally smaller in radius compared with star-forming galaxies at lower redshift with similar mass (Ferguson et al. 2004; Nagy et al. 2011; Law et al. 2012), but are larger than the compact ETGs, as seen in Figure 3. Despite this, our sample of compact candidate progenitors make up the low end of the size distribution of $z \sim 3$ LBGs. How do such small but massive galaxies form?

According to major merger simulations (see, e.g., Hopkins et al. 2008; Wuyts et al. 2010), with mass ratio from unity to $1: 10$, compact star-forming remnants may result from merging of galaxies with very large gas fractions, e.g., larger than $\sim 40 \%$. A compact remnant is formed when a sizable mass of new stars 
is formed at the center of the new structure by the highly dissipative gas. However, an extended stellar halo made by the older stellar populations will remain. Partners that are compact at the onset of the merging event, as well as a continuous accretion of gas as the merging progresses, increase the mass fraction of the remnant in the compact structure relative to that in the halo. According to the simulations, however, a sizable fraction of the stellar mass of the remnant will be found in the halo, in general disagreement with observations of compact ETGs (some have resorted to hiding the extended halo with dust obscuration to bring the simulations in better agreement with the observations (see, e.g., Wuyts et al. 2010)). In a simplified sense, gas-rich mergers produce two spatially segregated stellar populations: a centralized starburst, embedded in an extended older population.

A key feature of gas-rich merger remnants generally seen among simulations is the need for a two-component fit to the light profile: a centrally steeper one to account for the starburst component and a shallower, more extended one to account for the remnant stellar component (Hopkins et al. 2008; Wuyts et al. 2010; Bournaud et al. 2011). When fitting single Sersic profiles to simulated gas-rich mergers, Wuyts et al. (2010) find that the segregation of older and younger stellar populations, primarily driven by the combination of light excess of the dispersed older stellar populations at large radii and central cusp from the starburst, causes the Sersic fits to be better approximated with large values of the Sersic index, $n$. As a result, they find the following distribution of Sersic indices and effective radii from gas-rich merger simulations. Merger remnants are best fit by cuspy, high-Sersic index fits $(n>10)$, along with large radii (driven by the extended component) too large to be consistent with compact ETGs (Wuyts et al. 2010, their Figure 12(b)). Compared with observed properties of the ETG sample of van Dokkum et al. (2008), there appears to be some disagreement between the observed measurements and those measured from gas-rich merger simulations.

For comparison, we have constructed a similar plot (Figure 16) showing the distribution of Sersic indices as a function of effective radius for the candidate sample and also the ETGs of Cassata et al. (2013). We also plot the measured properties from the $H$-band stacks for the candidate sample and compact ETGs shown in Figure 13, as the stacks are more sensitive to the presence (if any) of extended low-surface brightness structures that would increase effective radius. These measurements of the light profiles of compact ETGs represent an improvement in sample size, and in the case of the stacks, sensitivity to deviations from a Sersic profile and low surface-brightness features, relative to the comparison made with van Dokkum et al. (2008).

The distribution of our measurements also do not coincide with the simulated gas-rich merger measurements in that our galaxies are well-fit by single Sersic profiles and do not show cuspy centers or extended light at large radii. We also see no evidence of these features in any of our stacks, with all light profiles being well fit by single Sersic profiles with $n \sim 2-4$, down to the noise limit in our stacks, and in general agreement with the distribution of measurements from individual galaxies. To the extent that the simulations represent real gas-rich mergers, our data of compact ETGs and compact candidates (including both individual Sersic fits as well as those of the stacks) appear inconsistent with the simulations. We note that the distribution of radii of the models in the top and middle panels of Figure 16 is mainly driven by the fact that Sersic models are poor fits
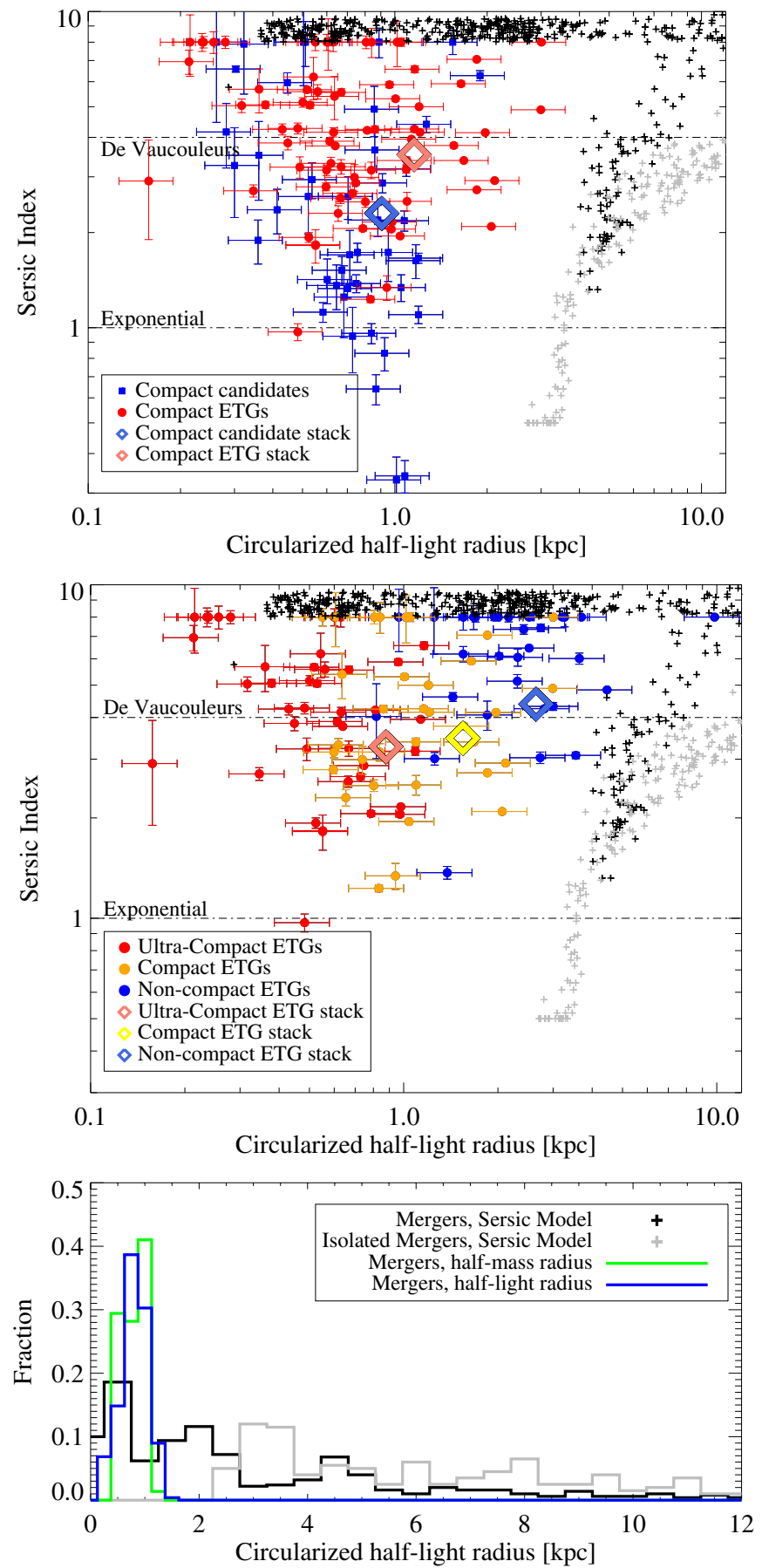

Figure 16. Top panel: distribution of Sersic index versus effective radius for candidates (blue) and compact ETGs (red). Measured values from the stacked images are shown in diamonds. Black and gray crosses represent the measurements of simulated gas-rich merger remnants and isolated mergers (isolated in the sense that the simulations do not include continuous gas inflow), respectively, from Wuyts et al. (2010, their Figure 12(b)). Middle panel: same plot with the Cassata et al. (2013) sample, split according to the compactness criteria. Bottom panel: the distribution of effective radii of the Wuyts et al. (2010) models presented earlier (black and gray), as modeled by Sersic profiles, along with direct measures of half-mass radii, half-light radii of the stellar component, and half-light radii after incorporating dust attenuation.

(A color version of this figure is available in the online journal.)

to the merger simulations. However, the actual simulated halfmass and half-light radii, without any assumption on the shape of the light profile, are still in agreement with the observations. This can be seen more clearly in the bottom panel, which shows 

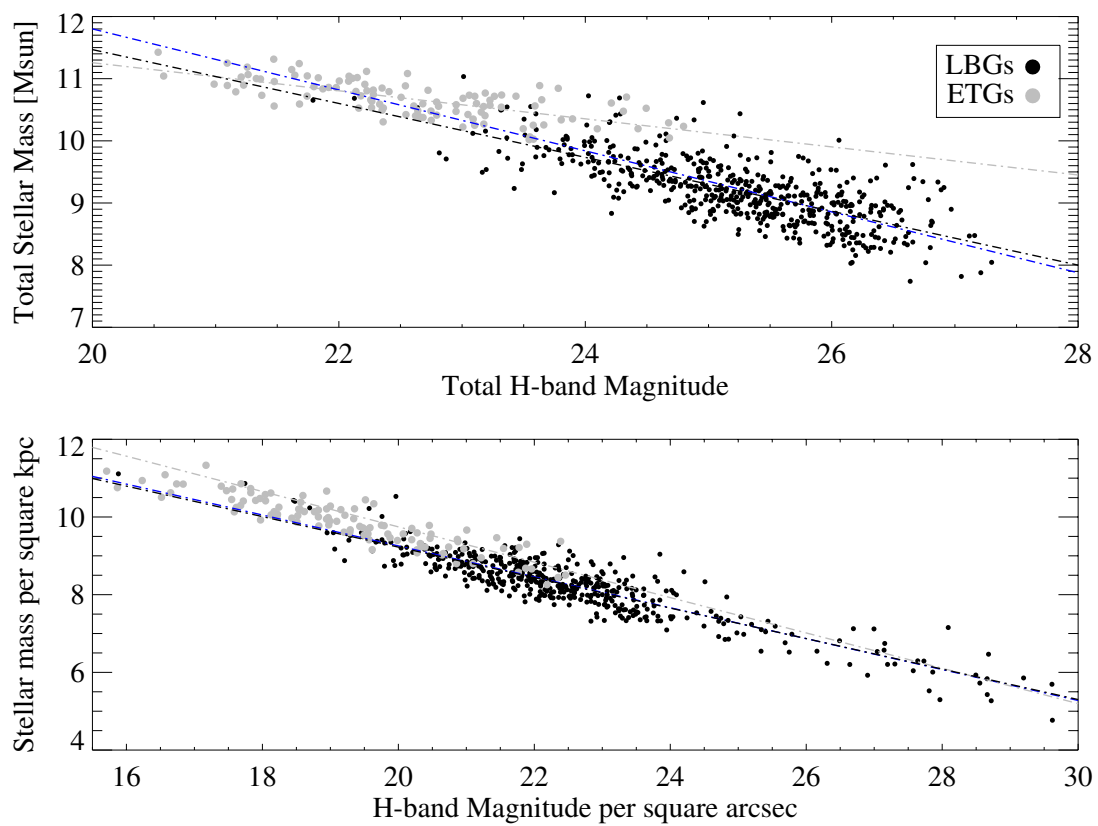

Figure 17. Top panel: correlation between the measured total $H$-band magnitude and total stellar mass for LBGs (black) and ETGs (gray). Linear fits to the two samples individually are shown in the same colors. A linear fit to all points together is shown in blue. Bottom panel: the correlation between the surface densities of these two quantities.

(A color version of this figure is available in the online journal.)

that typical half-mass and half-light radii of the simulations are on the order of $\sim 1 \mathrm{kpc}$. Therefore, we cannot rule out gas-rich merging on the basis of this analysis.

Major mergers are also characterized by tidal tails, debris, and other general disruptions of preexisting galaxy components. The timescales for dissipating these tidal features can be very long (1 Gyr), with longer timescales correlating with larger gas fractions (Lotz et al. 2010). Therefore, if gas-rich mergers are the primary producer of compact star-forming galaxies at high redshift, these features should still be present around our compact candidate sample. Since the stellar mass in tidal debris may make up a small fraction of total stellar mass (especially in the case of tidal debris), we estimate here exactly how much stellar mass in extended distributions our stacks account for, given the depth of the stack. This provides an upper limit to the amount of residual stellar mass that can be lurking undetected around our galaxy samples. To estimate this, we take advantage of the relationship between our measured total $H$-band magnitude and total stellar mass, shown in the top panel of Figure 17. This figure shows that the two are strongly correlated for all three samples (candidate LBGs, non-candidate LBGs, and compact ETGs) considered here, albeit with large scatter in the case of the total quantities for LBGs (top panel). Nevertheless, we can translate this relationship to magnitude per unit area and stellar mass per unit area to relate surface brightness with surface density of stellar mass. This relationship (for both samples of galaxies together) is shown in the bottom panel of Figure 17. Given the depths of our stacks, which go down to $\sim 29$ mag $\operatorname{arcsec}^{-2}$, we estimate that the stacks are sensitive to surface densities of at least $10^{6} M_{\odot} \mathrm{kpc}^{-2}$. This implies that if our candidate galaxy samples are compact because of major mergers, the progenitor(s) (i.e., the non-dissipational old stellar component) must contain less than this surface density of old stars in tidal debris or extended stellar halos.

Wuyts et al. (2010) present the range of surface brightness profiles of the extended stellar halos of gas-rich merger remnants, which are more extended than observed compact ETGs (their Figure 13). In Figure 18 we present a comparison of these simulated merger remnants, along with the intrinsic Sersic profile (i.e., unconvolved with the PSF) fit to the stacks of the LBG samples (top panel) as well as the ETG samples (bottom panel). We find that in all cases the intrinsic shape of the compact samples (candidate LBGs, compact, and ultra-compact ETGs) do not match the extended distributions of the simulated merger remnants. While the absolute normalization of the observed light profiles should depend on galaxy flux, distance, and mass, and are not necessarily expected to match the simulations in magnitude, the steepness and shape of the profiles on large scales (i.e., $1 \mathrm{kpc}<R<10 \mathrm{kpc}$ ) do not agree, independent of this normalization. In other words, the light profile of the compact galaxies is very often too steep relative to the extended distributions of the simulated merger remnants, suggesting that if this extended stellar halo were present in our samples, we would have detected it. This is in agreement with the comparisons made in Wuyts et al. (2010) to the observed ETGs of van Dokkum et al. (2008) and Szomoru et al. (2010), and we show that similar conclusions can be drawn for our candidate LBG sample. The extended stellar halo characteristic of simulated gas-rich merger remnants appears to be absent from our observed light profiles for compact galaxies. The steepness of the light profiles of these galaxies set important constraints that any mechanism of compact ETG creation must satisfy, and we have argued here that this excludes large disky galaxies with shallower profiles from being compact ETG progenitors.

\subsection{Speculation on a Possible Scenario for the Formation of the Compact ETGs and Compact Galaxies in General}

The aforementioned discussion proposes that gas-rich major merging of galaxies with a sizable stellar component, i.e., observable in the HST CANDELS images, is not the mechanism responsible for the formation of the massive, compact ETGs 

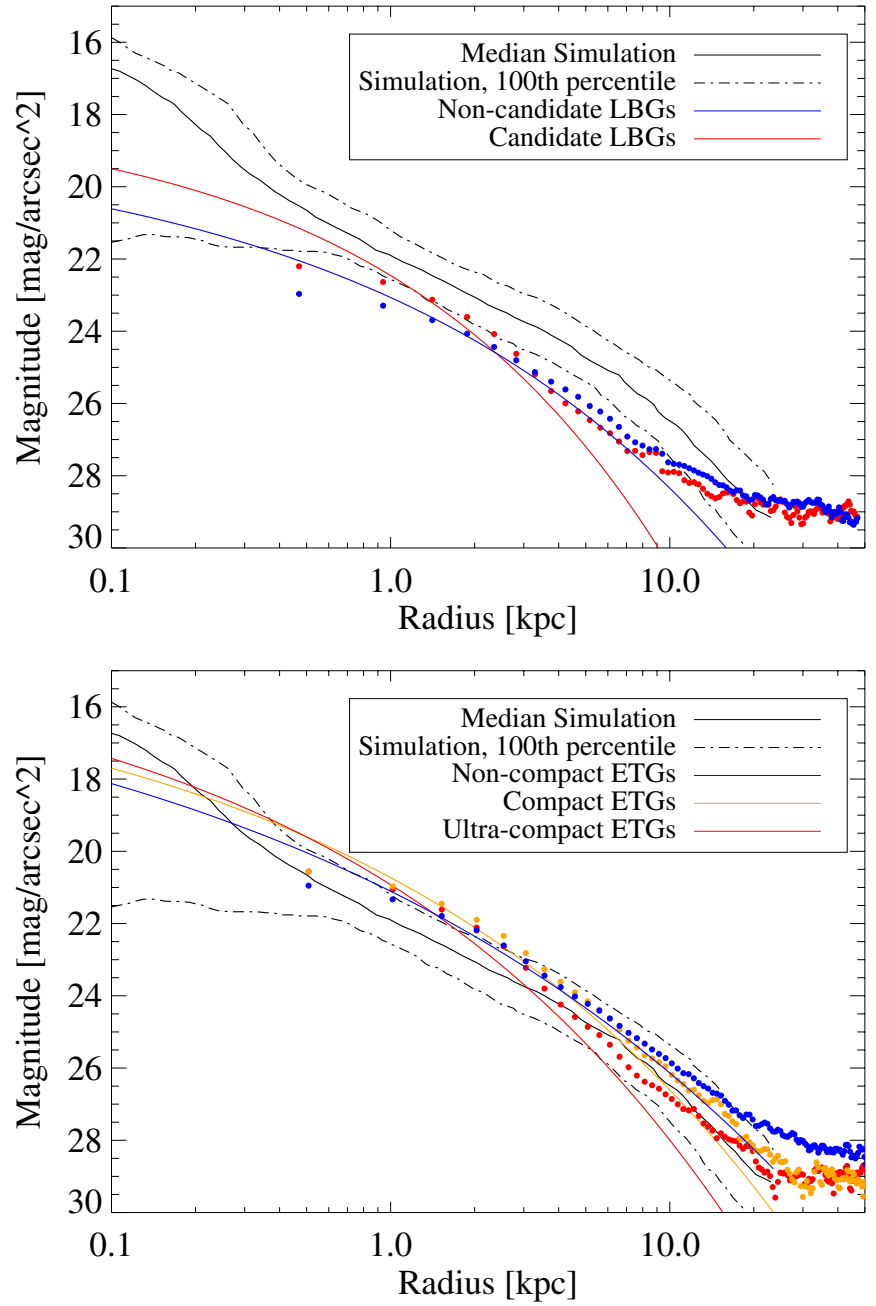

Figure 18. Top panel: intrinsic light profiles from the Wuyts et al. (2010) simulated gas-rich merger remnants (median and 100th percentile distributions) compared with the observed (points) and intrinsic (i.e., not convolved with PSF, lines) Sersic profiles of our LBG samples. Bottom panel: same as the top panel, but with the three samples of ETGs from Cassata et al. (2013).

(A color version of this figure is available in the online journal.)

observed at $\langle z\rangle=1.6$. We suggest that a more natural explanation of our observations may be that the star formation in these compact galaxies is being driven primarily by accretion of cold gas, which efficiently forms stars centrally rather than forming stars in an extended disk. The exact details of how the gas accretes have been discussed elsewhere by means of simulations and analytical calculations. It has been suggested that the main physical mechanism is one where the cold gas dissipates angular momentum in a compact disk (Danovich et al. 2012), and as more gas accretes, the disk develops VDI (Dekel et al. 2009a) that is very effective in driving the gas further down the bottom of the potential well, giving rise to a very compact structure. Direct cold mode accretion (CMA) of the cold gas into the compact structure is also another mechanism (Birnboim \& Dekel 2003; Kereš et al. 2005; Dekel \& Birnboim 2006) that can give rise to very compact star-forming galaxies (Johansson et al. 2012). Both the VDI and the CMA predict the formation of very compact star-forming galaxies, with the VDI-driven wet inflow predicting a mixture of a perturbed disk and a rotating compact bulge. It is important to keep in mind that current spectroscopic observations of both compact star-forming galaxies and passive galaxies (Onodera et al. 2012) at $z \sim 2$ do not have sufficient spatial resolution to distinguish between a compact disk and a spheroid (especially a rotating one), the kinematical signature of both structures simply being that of broadened emission and absorption lines. Regardless of the details of how the cold gas is funneled into very compact regions, the morphology of compact galaxies seems to require a highly dissipative mechanism for the assembly of their stellar mass as opposed to the merger of sub-galactic structures with a sizable preexisting stellar component.

Star formation in the compact galaxies is then subsequently quenched and they evolve passively afterward. Recent studies have shown that star formation can be quenched solely by feedback from the highly concentrated stellar distribution. Two examples are stellar winds driven by intense starbursts (Rupke et al. 2005; Tremonti et al. 2007; Heckman et al. 2011) and internal ram pressure on dust grains (Murray et al. 2005). These feedback mechanisms imprint a maximum possible surface density of star formation (Eddington limited) (Murray et al. 2005; Thompson et al. 2005; Hopkins et al. 2010) and evidence of this has been seen in extremely rare compact starbursts at lower redshifts (Diamond-Stanic et al. 2012). Other studies have shown that at high redshift, compactness is the most sensitive statistical predictor of passivity among massive galaxies (Bell et al. 2012), a fact that is in broad general agreement with the fact that compact and ultra-compact galaxies dominate the population of passive galaxies at $z>2$, and with the finding we have reported here that compact star-forming galaxies appear to have more evolved bursts compared with non-compact ones.

In conclusion, we speculate that the high-redshift compact ETGs are the direct descendants of compact, star-forming galaxies, which themselves are compact because their star formation is primarily driven by the accretion of cold gas to the central regions of the galaxy. Their star formation is quenched as a result of their compactness because of stellar feedback (e.g., Diamond-Stanic et al. 2012), halo quenching (in the most massive cases; Dekel \& Birnboim 2006; Kereš et al. 2005; Birnboim \& Dekel 2003), or some combination of both, and they evolve passively after. If they undergo merging and/or accretion, their compactness is altered and they may end up forming a more diffuse light profile, and if compact star-forming galaxies do not form anymore, then the number of compact passive galaxies keeps decreasing with cosmic time (see Cassata et al. 2013). This scenario is generally supported by the distribution of stellar populations we present in Figure 13, as well as studies of the main drivers of high-redshift star formation (Conselice et al. 2013). With this study, we have shown that (1) there are sufficient galaxies to supply the observed abundance of compact ETGs this way and (2) it is not implausible that compact ETGs may be the descendants of compact star-forming LBGs.

The high-redshift ETG sample of Cassata et al. (2011, 2013) also contains a fraction of ETGs that are non-compact, (i.e., of a size similar to local ellipticals). At the highest redshifts $(z>1.5)$, the fraction is tiny, but the number density of noncompact ETGs increases dramatically to the present (Cassata et al. 2011, 2013). Detailed high-resolution studies of local ellipticals have shown they are best described by multiple morphological components (Kormendy et al. 2009), even up to three and four Sersic components (Huang et al. 2013b), suggesting episodic periods of structural buildup. Other studies have proposed that the compact ETGs are the cores of local ellipticals, with stellar mass buildup occurring in an insideout fashion (Bezanson et al. 2009; Hopkins et al. 2009a; 
van Dokkum et al. 2010; Huang et al. 2013b). What is clear is that the sizes of ETGs increase dramatically over time, in part because newly formed ETGs appear with progressively larger sizes as the universe evolves (Cassata et al. 2011, 2013). We suggest that these non-compact $z>1$ ETGs may form from an independent evolutionary track to the compact ETGs, with non-compact ETGs the result of high-redshift (major) merging activity.

At $z \gtrsim 1$, the number density of non-compact ETGs in the Cassata et al. (2013) sample steadily increases with time, and has increased sufficiently to make up half of all $M>10^{10} M_{\odot}$ ETGs by $z \sim 1$. Incidentally, the number density of galaxies that are likely to be gas-rich mergers is similar to that of noncompact ETGs at $z>1.2$ (see Table 1 ). With a constant merger rate with redshift, and assuming each merger quenches the star formation (at least for the next Gyr or two before another merger rejuvenates star formation), it is qualitatively plausible that this steady increase in non-compact ETGs could be explained by a constant supply of mergers per unit time. At $z<1$, during the buildup of these non-compact ETGs, primarily dry mergers are found to account for both the assembly of the massive end $\left(M>10^{11} M_{\odot}\right)$ of the red sequence (van der Wel et al. 2009a; Robaina et al. 2010), as well as explain their morphologies as measured at low redshift (van der Wel et al. 2009b). Direct comparison of number densities to assess whether the actual number densities of mergers is sufficient to explain the increase in non-compact ETGs at $z>1$ will need to await accurate counts of mergers out to higher redshifts.

\section{SUMMARY}

We have demonstrated the existence of a significant population of compact LBGs, which have consistent SFH, stellar-mass densities, and co-moving volume densities with high-redshift compact ETGs (the sample of Cassata et al. 2013). We find the following.

1. These candidate progenitors of compact ETGs show distinct SED properties from the non-candidates, consistent with an older burst of SF, i.e., the burst appears to show evidence of fading.

2. Stacking from IR images are consistent with this interpretation and favor an older burst over an increased contribution from dust.

3. The average X-ray properties of the compact and noncompact ones are consistent with each other. One interpretation is that AGN activity has not influenced the selection of the candidates.

4. Structural properties of candidates and compact ETGs differ from predictions of gas-rich merger simulations, suggesting this is not the dominant mechanism producing compact star-forming galaxies and compact ETGs at high redshift.

5. We suggest that compact ETGs are formed primarily through the quenching of compact star-forming galaxies whose in situ star formation is driven by cold accretion from the IGM via VDI and CMA. We speculate that mergerdriven evolution may contribute to the non-compact ETG population at high redshift.

We thank the anonymous referee whose valuable suggestions have improved this paper significantly. M.G. and C.C.W. thank Alvio Renzini for many fruitful and inspiring conversations on this work. We thank Benjamin Magnelli and Ranga-Ram

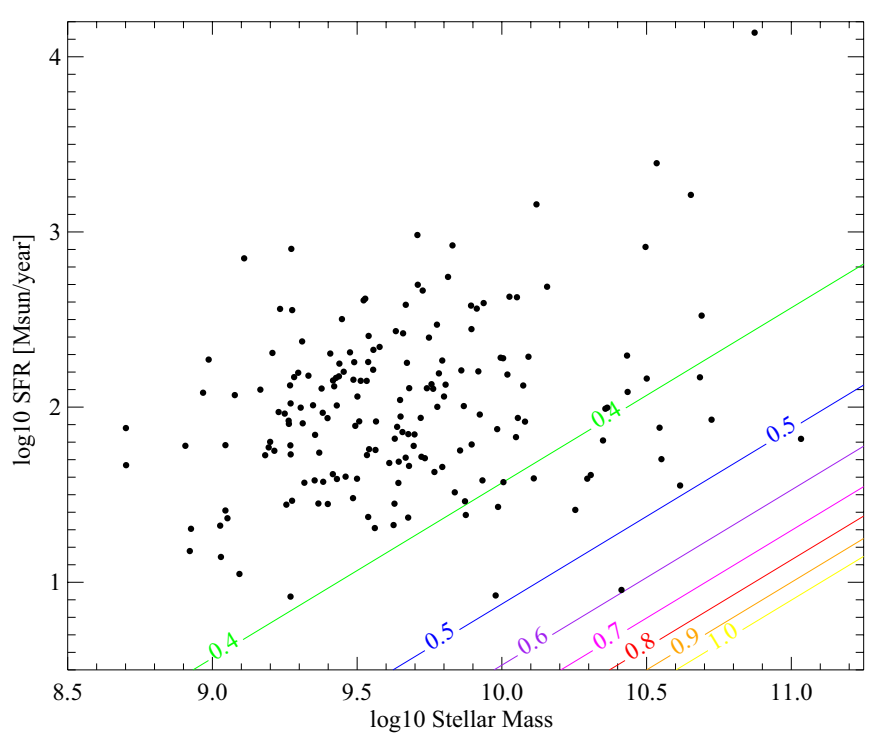

Figure 19. Limiting value of $\tau$ (in Gyr, contours) for exponentially declining SFHs. If the value of $\tau$ is larger than this limiting $\tau$ for a given stellar mass and SFR, then a galaxy at $z \sim 3$ will not meet the sSFR criteria of the ETG selection by $z \sim 1.6$. The majority of the parameter space occupied by our galaxies must have $\tau<0.4$ Gyr.

(A color version of this figure is available in the online journal.)

Chary for graciously providing $24 \mu \mathrm{m}$ residual maps for our stacking analysis. The study also benefited from illustrative discussions with Stacey Alberts, Alexandra Pope, and Arjen Van der Wel. C.C.W. thanks Joseph Meiring and Dan Popowich for technical consulting. This work is based on observations taken by the CANDELS Multi-Cycle Treasury Program with the NASA/ESA HST. We acknowledge support from grant program NSF AST 08-8133, and support for HST Program GO 12060.10-A was provided by NASA through grants from the Space Telescope Science Institute, which is operated by the Association of Universities for Research in Astronomy, Inc., under NASA contract NAS5-26555.

\section{APPENDIX}

\section{POSSIBLE QUENCHING PATHS FOR THE LBG}

We investigate first the general range of acceptable exponentially declining SFHs ( $\tau$-models) that agree with the red colors and low sSFRs of the compact ETGs. The low sSFRs of ETGs generally sets a limit to values of the decline timescale, $\tau$, and the time since the last starburst activity in the galaxy. Too recent a burst or too long a value of the decline timescale will leave too high of a sSFR in the galaxy. Because the sSFR is a function of SFR and stellar mass, we investigate how the limiting value of $\tau$ changes as a function of both SFR and stellar mass. In Figure 19, we show how large $\tau$ can be for a $z \sim 3$ galaxy, as a function of SFR and stellar mass measured at $z \sim 3$, and still have a measured sSFR $<-2 \mathrm{Gyr}^{-1}$ by $z \sim 1.6$. This figure shows that, for most LBGs in our sample (median $\mathrm{M}^{*}$ of $10^{9.5} M_{\odot}$, median SFR of $\sim 60 M_{\odot} \mathrm{yr}^{-1}$ ), the value of $\tau$ must be small ( $<400 \mathrm{Myr})$. A larger $\tau$ would result in non-negligible sSFR and exclusion of the galaxy by the compact ETG selection. It is not surprising that galaxies with lower initial sSFRs (bottom-right corner) can tolerate higher values of $\tau$ and still be considered passive by $z \sim 1.6$. 
We estimate a lower limit to $\tau$ according to the following assumption. For the star formation to decline in a galaxy, regardless of the quenching mechanism, the cold gas must be removed or depleted, and the shortest time in which this can happen is limited by the sound speed, $c_{s}$, in the ISM of a star-forming galaxy. In general, this assumption would imply that each LBG has its own quenching timescale related to the diameter of the galaxy, such that sound-crossing time (and therefore quenching time) is larger for larger galaxies. For small galaxies such as the more compact ones we consider here, this quenching time is relatively fast. For a typical sized LBG of a diameter $D \sim 2 \mathrm{kpc}$, we estimate the timescale $t=D / c_{s}=2 \mathrm{kpc} / 20 \mathrm{~km} \mathrm{~s}^{-1}=100 \mathrm{Myr}$ for cold gas depletion. In the analysis presented in this paper, we adopt this as our lower limit to $\tau$. However, to test the robustness against this assumption of a fixed minimum timescale for our sample, we note that repeating the analysis using a sample selected using a different value of $\tau$ for each galaxy that depends on size as outlined earlier does not significantly change our results.

With these constraints in hand, our strategy is to use what we do know about the SFHs of ETGs and work backward to gain some insight into which LBGs have consistent properties. We acknowledge that any given LBG may or may not follow an "ETG-consistent" SFH between $z=3$ and $z=1.6$, but note that any progenitors of ETGs among the LBGs must follow an "ETG-consistent" one. Therefore, the real ETG progenitors, if any exist among LBGs, will be contained in a sample selected this way.

We use the lower limit to $\tau$ calculated earlier to select our sample because it will result in the most conservative sample of plausible progenitors. By conservative, we mean that it results in the smallest number of candidates with the smallest increase in stellar masses over the course of $\sim 2$ Gyr. Repeating the analysis with a longer $\tau$ will cause only a net increase in stellar mass over this time, thus resulting in more massive galaxies and also adding more candidates. However, as Figure 19 suggests, alternative SFHs with slightly larger $\tau$ s are consistent with the observed compact ETG properties and so the effect of this assumption should be considered (although the range in allowable values of $\tau$ is small).

Phrased in another way, this range in $\tau$ introduces a scatter in the actual amount of stellar mass that LBGs will form (compared with that which we assume) between $z \sim 3$ and $z \sim 1.6$. In Figure 20, we assess exactly how much this scatter in stellar-mass buildup is between our upper and lower limits in $\tau$. To quantify the scatter, we calculate $(\Delta M(\tau) / M)=$ $\left(M\left(\tau_{\max }\right)-M(100 \mathrm{Myr}) / M\left(\tau_{\max }\right)\right)$ for a range of initial values of SFR and $M_{*}$ measured at $z \sim 3$, analogous to Figure 19. For each region of the figure, we use as the maximum value of $\tau$ the value derived in Figure 19. We find that the assumption of different $\tau$ values within the limits have less than a factor of two effect on the change in mass compared with final mass of the LBGs. We can compare these values to the actual uncertainty in the estimate of stellar mass in LBGs, for example, according to the simulations of stellar mass estimates for LBGs in the analysis of Lee et al. (2009). The estimated error in stellar mass of LBGs is magnitude dependent, but may vary up to a factor of two for $U$-band dropouts. The scatter we find in final mass, depending on assumption of the SFH, is less than the scatter associated with the initial LBG mass estimate. Therefore, the differences in extrapolated mass buildup from varying the assumed value of $\tau$ are not the dominant source of uncertainty in whether or not an LBG is included in the candidate sample. We also note that
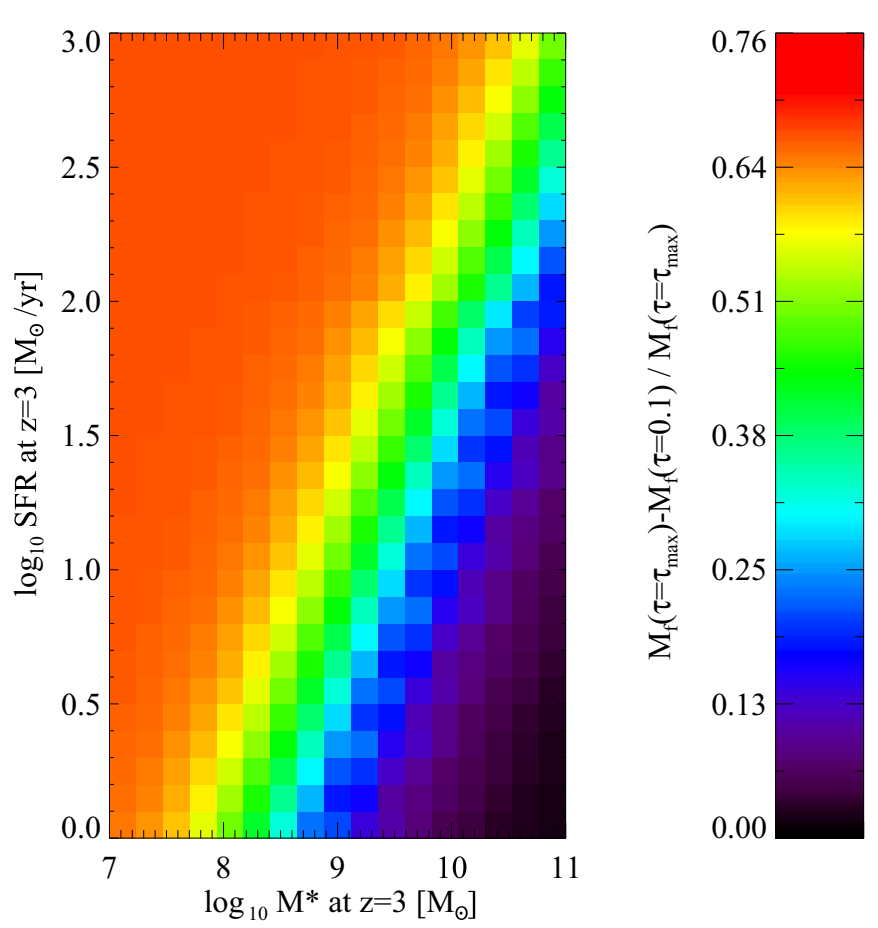

Figure 20. Difference in accumulated stellar mass between our assumed value of $\tau$ in Section 3 and the maximum $\tau$ allowable in Figure 19, compared with the maximum amount. The difference between the two is somewhat comparable to the uncertainties in stellar mass measurements in Lee et al. (2009).

(A color version of this figure is available in the online journal.)

we do not make an attempt to incorporate mass-loss from stars that are returned to the ISM during the cycle of star-formation.

\section{REFERENCES}

Barnes, J. E. 1992, ApJ, 393, 484

Barro, G., Faber, S. M., Pérez-González, P. G., et al. 2013, ApJ, 765, 104 Bell, E. F., Phleps, S., Somerville, R. S., et al. 2006, ApJ, 652, 270 Bell, E. F., van der Wel, A., Papovich, C., et al. 2012, ApJ, 753, 167 Bezanson, R., van Dokkum, P. G., Tal, T., et al. 2009, ApJ, 697, 1290 Birnboim, Y., \& Dekel, A. 2003, MNRAS, 345, 349

Blain, A. W., Chapman, S. C., Smail, I., \& Ivison, R. 2004, ApJ, 611, 725 Bouché, N., Cresci, G., Davies, R., et al. 2007, ApJ, 671, 303 Bournaud, F., Chapon, D., Teyssier, R., et al. 2011, ApJ, 730, 4 Bournaud, F., Jog, C. J., \& Combes, F. 2007, A\&A, 476, 1179 Brodwin, M., Dey, A., Brown, M. J. I., et al. 2008, ApJL, 687, L65 Bruzual, G. 2007, in ASP Conf. Ser. 374, From Stars to Galaxies: Building the Pieces to Build Up the Universe, ed. A. Vallenari, R. Tantalo, L. Portinari, \& A. Moretti (San Francisco, CA: ASP), 303

Bruzual, G., \& Charlot, S. 2003, MNRAS, 344, 1000

Bundy, K., Ellis, R. S., Conselice, C. J., et al. 2006, ApJ, 651, 120

Bundy, K., Fukugita, M., Ellis, R. S., et al. 2009, ApJ, 697, 1369

Bundy, K., Treu, T., \& Ellis, R. S. 2007, ApJL, 665, L5

Bussmann, R. S., Gurwell, M. A., Fu, H., et al. 2012, ApJ, 756, 134

Calzetti, D., Armus, L., Bohlin, R. C., et al. 2000, ApJ, 533, 682

Carollo, C. M., Bschorr, T. J., Renzini, A., et al. 2013, ApJ, 773, 112

Cassata, P., Giavalisco, M., Guo, Y., et al. 2011, ApJ, 743, 96

Cassata, P., Giavalisco, M., Williams, C. C., et al. 2013, ApJ, 775, 106

Chapman, S. C., Blain, A. W., Smail, I., \& Ivison, R. J. 2005, ApJ, 622, 772

Chary, R., \& Elbaz, D. 2001, ApJ, 556, 562

Cheung, E., Faber, S. M., Koo, D. C., et al. 2012, ApJ, 760, 131

Cimatti, A., Cassata, P., Pozzetti, L., et al. 2008, A\&A, 482, 21

Cimatti, A., Daddi, E., \& Renzini, A. 2006, A\&A, 453, L29

Conselice, C. J., Mortlock, A., Bluck, A. F. L., Grützbauch, R., \& Duncan, K. 2013, MNRAS, 430, 1051

Cristiani, S., Appenzeller, I., Arnouts, S., et al. 2000, A\&A, 359, 489

Daddi, E., Dannerbauer, H., Stern, D., et al. 2009, ApJ, 694, 1517

Daddi, E., Renzini, A., Pirzkal, N., et al. 2005, ApJ, 626, 680

Damjanov, I., McCarthy, P. J., Abraham, R. G., et al. 2009, ApJ, 695, 101 
Danovich, M., Dekel, A., Hahn, O., \& Teyssier, R. 2012, MNRAS, 422, 1732 Dekel, A., \& Birnboim, Y. 2006, MNRAS, 368, 2

Dekel, A., \& Burkert, A. 2013, arXiv:1310.1074D

Dekel, A., Birnboim, Y., Engel, G., et al. 2009a, Natur, 457, 451

Dekel, A., Sari, R., \& Ceverino, D. 2009b, ApJ, 703, 785

Dekel, A., Zolotov, A., Tweed, D., et al. 2013, MNRAS, 435, 999

Diamond-Stanic, A. M., Moustakas, J., Tremonti, C. A., et al. 2012, ApJL, 755, L26

Elbaz, D., Dickinson, M., Hwang, H. S., et al. 2011, A\&A, 533, A119

Engelbracht, C. W., Blaylock, M., Su, K. Y. L., et al. 2007, PASP, 119, 994

Faber, S. M., \& Jackson, R. E. 1976, ApJ, 204, 668

Ferguson, H. C., Dickinson, M., Giavalisco, M., et al. 2004, ApJL, 600, L107

Fioc, M., \& Rocca-Volmerange, B. 1997, A\&A, 326, 950

Forbes, J. C., Krumholz, M. R., Burkert, A., \& Dekel, A. 2013, arXiv:1311.1509

Genel, S., Bouché, N., Naab, T., Sternberg, A., \& Genzel, R. 2010, ApJ, 719,229

Giavalisco, M. 2002, ARA\&A, 40, 579

Giavalisco, M., Ferguson, H. C., Koekemoer, A. M., et al. 2004, ApJL, 600, L93

Grogin, N. A., Kocevski, D. D., Faber, S. M., et al. 2011, ApJS, 197, 35

Guo, Y., Ferguson, H. C., Giavalisco, M., et al. 2013, ApJS, 207, 24

Guo, Y., Giavalisco, M., Cassata, P., et al. 2012, ApJ, 749, 149

Hathi, N. P., Cohen, S. H., Ryan, R. E. Jr., et al. 2013, ApJ, 765, 88

Hathi, N. P., Jansen, R. A., Windhorst, R. A., et al. 2008a, AJ, 135, 156

Hathi, N. P., Malhotra, S., \& Rhoads, J. E. 2008b, ApJ, 673, 686

Heckman, T. M., Borthakur, S., Overzier, R., et al. 2011, ApJ, 730, 5

Hernquist, L. 1992, ApJ, 400, 460

Hernquist, L. 1993, ApJ, 409, 548

Hickox, R. C., Wardlow, J. L., Smail, I., et al. 2012, MNRAS, 421, 284

Hopkins, P. F., Bundy, K., Murray, N., et al. 2009a, MNRAS, 398, 898

Hopkins, P. F., Cox, T. J., Dutta, S. N., et al. 2009b, ApJS, 181, 135

Hopkins, P. F., Hernquist, L., Cox, T. J., Dutta, S. N., \& Rothberg, B. 2008, ApJ, 679,156

Hopkins, P. F., Hernquist, L., Cox, T. J., et al. 2006, ApJS, 163, 1

Hopkins, P. F., Murray, N., Quataert, E., \& Thompson, T. A. 2010, MNRAS, 401, L19

Huang, K.-H., Ferguson, H. C., Ravindranath, S., \& Su, J. 2013a, ApJ, 765, 68 Huang, S., Ho, L. C., Peng, C. Y., Li, Z.-Y., \& Barth, A. J. 2013b, ApJ, 766, 47 Ilbert, O., Salvato, M., Le Floc'h, E., et al. 2010, ApJ, 709, 644

Johansson, P. H., Naab, T., \& Ostriker, J. P. 2012, ApJ, 754, 115

Kartaltepe, J. S., Dickinson, M., Alexander, D. M., et al. 2012, ApJ, 757, 23

Kartaltepe, J. S., Sanders, D. B., Le Floc'h, E., et al. 2010, ApJ, 721, 98

Kaviraj, S., Cohen, S., Ellis, R. S., et al. 2013a, MNRAS, 428, 925

Kaviraj, S., Cohen, S., Windhorst, R. A., et al. 2013b, MNRAS, 429, L40

Kereš, D., Katz, N., Fardal, M., Davé, R., \& Weinberg, D. H. 2009, MNRAS, 395,160

Kereš, D., Katz, N., Weinberg, D. H., \& Davé, R. 2005, MNRAS, 363, 2

Khochfar, S., \& Silk, J. 2006, ApJL, 648, L21

Koekemoer, A. M., Faber, S. M., Ferguson, H. C., et al. 2011, ApJS, 197, 36

Kormendy, J., Fisher, D. B., Cornell, M. E., \& Bender, R. 2009, ApJS, 182, 216

Kurk, J., Cimatti, A., Daddi, E., et al. 2009, A\&A, 504, 331

Laidler, V. G., Papovich, C., Grogin, N. A., et al. 2007, PASP, 119, 1325

Law, D. R., Steidel, C. C., Shapley, A. E., et al. 2012, ApJ, 745, 85

Lee, B., Giavalisco, M., Williams, C. C., et al. 2013, ApJ, 774, 47

Lee, K.-S., Dey, A., Reddy, N., et al. 2011, ApJ, 733, 99

Lee, S.-K., Ferguson, H. C., Somerville, R. S., Wiklind, T., \& Giavalisco, M. 2010, ApJ, 725, 1644

Lee, K.-S., Ferguson, H. C., Wiklind, T., et al. 2012, ApJ, 752, 66

Lee, S.-K., Idzi, R., Ferguson, H. C., et al. 2009, ApJS, 184, 100

Le Fèvre, O., Vettolani, G., Paltani, S., et al. 2004, A\&A, 428, 1043

Lilly, S. J., Eales, S. A., Gear, W. K. P., et al. 1999, ApJ, 518, 641

López-Sanjuan, C., Le Fèvre, O., Ilbert, O., et al. 2012, A\&A, 548, A7

Lotz, J. M., Davis, M., Faber, S. M., et al. 2008, ApJ, 672, 177

Lotz, J. M., Jonsson, P., Cox, T. J., \& Primack, J. R. 2010, MNRAS, 404, 590

Lutz, D., Poglitsch, A., Altieri, B., et al. 2011, A\&A, 532, A90

Madau, P. 1995, ApJ, 441, 18

Madau, P., Pozzetti, L., \& Dickinson, M. 1998, ApJ, 498, 106

Magnelli, B., Elbaz, D., Chary, R. R., et al. 2011, A\&A, 528, A35

Marchesini, D., Whitaker, K. E., Brammer, G., et al. 2010, ApJ, 725, 1277

Martig, M., Bournaud, F., Teyssier, R., \& Dekel, A. 2009, ApJ, 707, 250

McLure, R. J., Pearce, H. J., Dunlop, J. S., et al. 2013, MNRAS, 428, 1088

Meurer, G. R., Heckman, T. M., \& Calzetti, D. 1999, ApJ, 521, 64

Mignoli, M., Cimatti, A., Zamorani, G., et al. 2005, A\&A, 437, 883

Mosleh, M., Williams, R. J., Franx, M., \& Kriek, M. 2011, ApJ, 727, 5

Murray, N., Quataert, E., \& Thompson, T. A. 2005, ApJ, 618, 569

Muzzin, A., Marchesini, D., Stefanon, M., et al. 2013, ApJ, 777, 18

Naab, T., Johansson, P. H., \& Ostriker, J. P. 2009, ApJL, 699, L178
Naab, T., Johansson, P. H., Ostriker, J. P., \& Efstathiou, G. 2007, ApJ, 658, 710 Nagy, S. R., Law, D. R., Shapley, A. E., \& Steidel, C. C. 2011, ApJL, 735, L19 Newman, A. B., Ellis, R. S., Bundy, K., \& Treu, T. 2012, ApJ, 746, 162

Nipoti, C., Treu, T., Leauthaud, A., et al. 2012, MNRAS, 422, 1714

Nonino, M., Dickinson, M., Rosati, P., et al. 2009, ApJS, 183, 244

Onodera, M., Renzini, A., Carollo, M., et al. 2012, ApJ, 755, 26

Oogi, T., \& Habe, A. 2013, MNRAS, 428, 641

Oser, L., Naab, T., Ostriker, J. P., \& Johansson, P. H. 2012, ApJ, 744, 63

Oser, L., Ostriker, J. P., Naab, T., Johansson, P. H., \& Burkert, A. 2010, ApJ, 725,2312

Ostriker, J. P. 1980, ComAp, 8, 177

Ownsworth, J. R., Conselice, C. J., Mortlock, A., Hartley, W. G., \&

Buitrago, F. 2012, MNRAS, 426, 764

Patel, S. G., van Dokkum, P. G., Franx, M., et al. 2013, ApJ, 766, 15

Peng, C. Y., Ho, L. C., Impey, C. D., \& Rix, H.-W. 2002, AJ, 124, 266

Peng, Y.-j., Lilly, S. J., Kovač, K., et al. 2010, ApJ, 721, 193

Peng, Y.-j., Lilly, S. J., Renzini, A., \& Carollo, M. 2012, ApJ, 757, 4

Poggianti, B. M., Calvi, R., Bindoni, D., et al. 2013, ApJ, 762, 77

Popesso, P., Dickinson, M., Nonino, M., et al. 2009, A\&A, 494, 443

Pozzetti, L., Bolzonella, M., Zucca, E., et al. 2010, A\&A, 523, A13

Ragone-Figueroa, C., \& Granato, G. L. 2011, MNRAS, 414, 3690

Ravikumar, C. D., Puech, M., Flores, H., et al. 2007, A\&A, 465, 1099

Ravindranath, S., Giavalisco, M., Ferguson, H. C., et al. 2006, ApJ, 652, 963

Renzini, A. 2009, MNRAS, 398, L58

Retzlaff, J., Rosati, P., Dickinson, M., et al. 2010, A\&A, 511, A50

Robaina, A. R., Bell, E. F., van der Wel, A., et al. 2010, ApJ, 719, 844

Rupke, D. S., Veilleux, S., \& Sanders, D. B. 2005, ApJS, 160, 115

Ryan, R. E. Jr., McCarthy, P. J., Cohen, S. H., et al. 2012, ApJ, 749, 53

Sales, L. V., Navarro, J. F., Theuns, T., et al. 2012, MNRAS, 423, 1544

Sanders, D. B., \& Mirabel, I. F. 1996, ARA\&A, 34, 749

Sanders, D. B., Soifer, B. T., Elias, J. H., et al. 1988, ApJ, 325, 74

Saracco, P., Gargiulo, A., \& Longhetti, M. 2012, MNRAS, 422, 3107

Saracco, P., Longhetti, M., \& Andreon, S. 2009, MNRAS, 392, 718

Saracco, P., Longhetti, M., \& Gargiulo, A. 2010, MNRAS, 408, L21

Saracco, P., Longhetti, M., \& Gargiulo, A. 2011, MNRAS, 412, 2707

Scott, S. E., Fox, M. J., Dunlop, J. S., et al. 2002, MNRAS, 331, 817

Scranton, R., Johnston, D., Dodelson, S., et al. 2002, ApJ, 579, 48

Smail, I., Chapman, S. C., Blain, A. W., \& Ivison, R. J. 2004, ApJ, 616, 71

Springel, V., Di Matteo, T., \& Hernquist, L. 2005, ApJL, 620, L79

Stefanon, M., Marchesini, D., Rudnick, G. H., Brammer, G. B., \& Whitaker, K.

E. 2013, ApJ, 768, 92

Steidel, C. C., \& Hamilton, D. 1993, AJ, 105, 2017

Swinbank, A. M., Chapman, S. C., Smail, I., et al. 2006, MNRAS, 371, 465

Swinbank, A. M., Smail, I., Chapman, S. C., et al. 2010, MNRAS, 405, 234

Szokoly, G. P., Bergeron, J., Hasinger, G., et al. 2004, ApJS, 155, 271

Szomoru, D., Franx, M., \& van Dokkum, P. G. 2012, ApJ, 749, 121

Szomoru, D., Franx, M., van Dokkum, P. G., et al. 2010, ApJL, 714, L244

Tacconi, L. J., Genzel, R., Smail, I., et al. 2008, ApJ, 680, 246

Tacconi, L. J., Neri, R., Chapman, S. C., et al. 2006, ApJ, 640, 228

Targett, T. A., Dunlop, J. S., Cirasuolo, M., et al. 2013, MNRAS, 432, 2012

Targett, T. A., Dunlop, J. S., McLure, R. J., et al. 2011, MNRAS, 412, 295

Taylor, E. N., Franx, M., Glazebrook, K., et al. 2010, ApJ, 720, 723

Thompson, T. A., Quataert, E., \& Murray, N. 2005, ApJ, 630, 167

Tremonti, C. A., Moustakas, J., \& Diamond-Stanic, A. M. 2007, ApJL, 663, L77

Trujillo, I., Conselice, C. J., Bundy, K., et al. 2007, MNRAS, 382, 109

Trujillo, I., Feulner, G., Goranova, Y., et al. 2006, MNRAS, 373, L36

Valentinuzzi, T., Fritz, J., Poggianti, B. M., et al. 2010a, ApJ, 712, 226

Valentinuzzi, T., Poggianti, B. M., Saglia, R. P., et al. 2010b, ApJL, 721, L19

van der Wel, A., Bell, E. F., van den Bosch, F. C., Gallazzi, A., \& Rix, H.-W. 2009a, ApJ, 698, 1232

van der Wel, A., Holden, B. P., Zirm, A. W., et al. 2008, ApJ, 688, 48

van der Wel, A., Rix, H.-W., Holden, B. P., Bell, E. F., \& Robaina, A. R.

2009b, ApJL, 706, L120

van Dokkum, P. G., Franx, M., Kriek, M., et al. 2008, ApJL, 677, L5

van Dokkum, P. G., Whitaker, K. E., Brammer, G., et al. 2010, ApJ, 709, 1018

Vanzella, E., Cristiani, S., Dickinson, M., et al. 2005, A\&A, 434, 53

Vanzella, E., Cristiani, S., Dickinson, M., et al. 2008, A\&A, 478, 83

Weinzirl, T., Jogee, S., Conselice, C. J., et al. 2011, ApJ, 743, 87

Whitaker, K. E., Kriek, M., van Dokkum, P. G., et al. 2012, ApJ, 745, 179

Whitaker, K. E., van Dokkum, P. G., Brammer, G., et al. 2010, ApJ, 719, 1715

Williams, C. C., Giavalisco, M., Porciani, C., et al. 2011a, ApJ, 733, 92

Williams, R. J., Quadri, R. F., \& Franx, M. 2011b, ApJL, 738, L25

Williams, R. J., Quadri, R. F., Franx, M., et al. 2010, ApJ, 713, 738

Windhorst, R. A., Cohen, S. H., Hathi, N. P., et al. 2011, ApJS, 193, 27

Wuyts, S., Cox, T. J., Hayward, C. C., et al. 2010, ApJ, 722, 1666

Xue, Y. Q., Luo, B., Brandt, W. N., et al. 2011, ApJS, 195, 10 